\title{
Acute myeloid leukemia: current progress and future directions
}

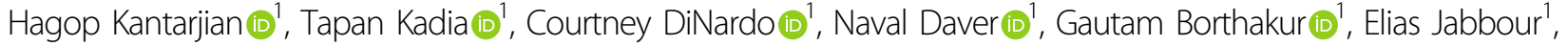 \\ Guillermo Garcia-Manero (D)', Marina Konopleva (D) and Farhad Ravandi ${ }^{1}$
}

\begin{abstract}
Progress in the understanding of the biology and therapy of acute myeloid leukemia (AML) is occurring rapidly. Since 2017, nine agents have been approved for various indications in AML. These included several targeted therapies like venetoclax, FLT3 inhibitors, IDH inhibitors, and others. The management of AML is complicated, highlighting the need for expertise in order to deliver optimal therapy and achieve optimal outcomes. The multiple subentities in AML require very different therapies. In this review, we summarize the important pathophysiologies driving AML, review current therapies in standard practice, and address present and future research directions.
\end{abstract}

\section{Introduction}

Progress in understanding the pathophysiology and improving the therapy of acute myeloid leukemia (AML) is now occurring at a rapid pace. The discovery of the activity of cytarabine (ara-C) and of anthracyclines in AML, and combining them in the 1970's, into what is known as the " $3+7$ regimen" ( 3 days of daunorubicin + 7 days of cytarabine), has long been considered the standard of care, resulting in long-term cures of 30 to $40 \%$ among younger patients with $A M L^{1-5}$. The earlier studies focused on patients usually up to the age of $50-55$ years, and reported 5-year survival rates of $40-45 \%$. Later studies including patients up to the age of 60 years reported 5 -year survival rates of $30-35 \%$. These intensive chemotherapy regimens, applied commonly in older patients (age 60 years and older), resulted in 5-year survival rates of $<10-15 \%^{6,7}$. Figure 1 shows the MD Anderson outcomes in AML in younger and older patients from 1970 to 2018 .

Unraveling the heterogeneity of AML at the clinical, cytogenetic, and molecular levels allowed improved prognostic and predictive abilities and led to the development of selected therapies for AML subsets. Chemotherapy-free

Correspondence: Hagop Kantarjian (hkantarjian@mdanderson.org)

${ }^{1}$ Department of Leukemia, MD Anderson Cancer Center, Houston, TX, USA regimens consisting of all trans-retinoic acid (ATRA) and arsenic trioxide in acute promyelocytic leukemia (APL) resulted in cure rates of $90 \%{ }^{8-12}$. In core-binding factor (CBF) AML, adding gemtuzumab ozogamicin (CD33-targeted monoclonal antibody conjugated to the calicheamicin payload) to high-dose cytarabine-based chemotherapy increased the long-term survival rate from $50 \%$ to $75+\%{ }^{13-17}$.

Research efforts in the last decade have expanded the pathophysiologic-molecular subsets of AML, through identification of prognostic, predictive, and targetable molecular abnormalities ${ }^{18-25}$. Ongoing studies and recently approved agents in AML of particular interest include: (1) Combinations of epigenetic therapy with hypomethylating agents (HMAs; azacitidine, decitabine) and venetoclax in older patients (or patients unfit for intensive chemotherapy); and combinations of intensive chemotherapy and venetoclax in younger/fit patients. (2) The addition of fms-like tyrosine kinase 3 (FLT3) inhibitors (gilteritinib, midostaurin, sorafenib, quizartinib, crenolanib, others) to intensive chemotherapy, or to HMA/low-intensity therapy in FLT3-mutated AML. (3) The addition of IDH inhibitors (IDH1 inhibitor ivosidenib; IDH2 inhibitor enasidenib) and/or venetoclax in IDH1/2- mutated AML. (4) Investigations of the roles of APR246 (TP53 modulator) and of magrolimab (antiCD47 monoclonal antibody enhancing the macrophage- 

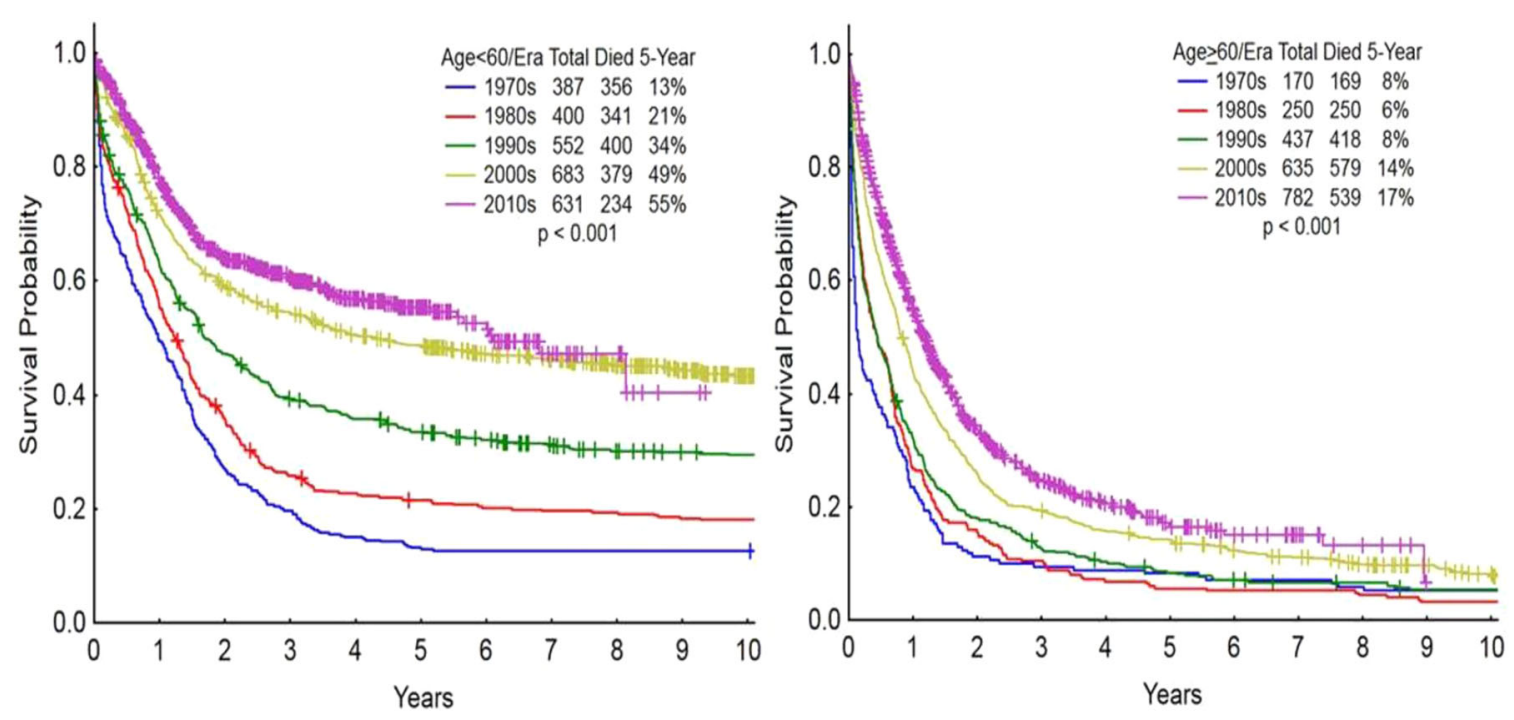

Fig. 1 Survival of de novo acute myeloid leukemia at MD Anderson (1970-2017) by Age and Treatment Era: Left panel: age<60 years; Right panel: age $60+$ years.

mediated phagocytosis) in TP53-mutated AML. (5) Exploring the role of menin inhibitors in mixed-lineage leukemia (MLL1)-rearranged acute leukemia. (6) Investigations of combined small-molecule targeted therapies, with or without standard intensive chemotherapy or HMAs (+/- venetoclax; at the expense of worsening myelosuppression), in order not only to prolong survival, but also to improve the potential cure rates in previously incurable AML subsets. (7) Establishing maintenance therapy as an important strategy in AML (as it is in acute lymphoblastic leukemia [ALL]). (8) Developing oral antiAML therapy (e.g., oral decitabine, oral azacitidine) to replace and improve upon the effects of parenteral therapies. (9) Approaches to enhance T-cell immune responses to AML (as done in ALL) with T-cell engagers (BiTEs), checkpoint inhibitors, and chimeric antigen receptor (CAR)-T-cell approaches.

Many AML experts ascribe to the $3+7$ regimen as the AML standard of care today; others may not. We will discuss the results with $3+7$ and put them into context with the more recent combined modality regimens, which may be superior. The nihilistic mood that prevailed in the AML community until 2015 has lifted, particularly with research resulting in the FDA approvals of multiple agents for AML since 2017 (Table 1). It is interesting to compare this AML review to the one published in $2016^{1}$, to appreciate the previous "bare cupboard" in AML research and the tremendous progress over such a short period of time. Prior to 2017, some decisions may have temporarily slowed progress in AML. One example is the voluntary withdrawal of gemtuzumab ozogamicin (GO) by the manufacturer (June 2010) from clinical use in the United
States based on a negative trial by the Southwest Oncology Group (SWOG) ${ }^{16}$. This was remedied with the GO re-approval in 2017 at a lower dose to minimize toxicity, based on a meta-analysis of five randomized frontline trials in AML clearly demonstrating benefit ${ }^{17}$. The use of $\mathrm{GO}$ is now particularly important in the therapy of $\mathrm{CBF}$ AML and APL. The second example was the nonapproval of decitabine in the US for frontline therapy of older patients with AML (approved in Europe ${ }^{26,27}$. Lowintensity HMA therapy with decitabine and azacitidinebased regimens is now the most common form of treatment among older (or unfit for intensive chemotherapy) patients with $\mathrm{AML}^{26,28}$. A third possible example is the non-submission of vosaroxin for FDA approval for the therapy of AML first salvage ${ }^{29}$. Vosaroxin may have offered a non-cardiotoxic form of topoisomerase-II inhibitor therapy.

In this review, we discuss progress in AML research, outline the MD Anderson approaches in 2020, and explore investigational strategies over the coming years.

\section{Cytogenetic and molecular abnormalities}

Acute myeloid leukemia has diverged from being considered as one acute leukemia entity to become a heterogeneous constellation of AML subentities characterized by diverse pathophysiologic, clinical, cytogenetic, and molecular profiles that benefit from individualized selective therapies and have vastly different outcomes.

The cytogenetic-molecular entities in AML are outlined in Table $2^{30-50}$. These include APL with its characteristic translocation 15;17 [t(15;17) (q22,q21)]; inversion 16 [inv 
Table 1 Recent Food and Drug Administration Drug Approvals (since 2017) in acute myeloid leukemia.

\begin{tabular}{|c|c|c|}
\hline Treatment (approval date) & Description & Indication \\
\hline Midostaurin (April 2017) & Multikinase FLT3 inhibitor & $\begin{array}{l}\text { Newly diagnosed FLT3-mutated (as detected by FDA-approved test) } \\
\text { AML, in combination with standard cytarabine and daunorubicin } \\
\text { induction and cytarabine consolidation }\end{array}$ \\
\hline $\begin{array}{l}\text { Gemtuzumab ozogamycin } \\
\text { (September 2017) }\end{array}$ & Anti-CD33 antibody-drug conjugate & $\begin{array}{l}\text { Adults with newly diagnosed CD33-positive AML; refractory-relapsed } \\
\text { CD33-positive } A M L \text { in patients } \geq 2 \text { years of age }\end{array}$ \\
\hline CPX-351 (August 2017) & $\begin{array}{l}\text { Liposomal cytarabine and daunorubicin } \\
\text { at a fixed 5:1 molar ratio }\end{array}$ & $\begin{array}{l}\text { Newly diagnosed therapy-related } \mathrm{AML} \text {, secondary } \mathrm{AML} \text { or } \mathrm{AML} \text { with } \\
\text { myelodysplasia-related changes }\end{array}$ \\
\hline Glasdegib (November 2018) & Hedgehog pathway inhibitor & $\begin{array}{l}\text { Newly diagnosed AML aged } \geq 75 \text { years or with co-morbidities that } \\
\text { preclude the use of intensive induction chemotherapy (in } \\
\text { combination with low-dose cytarabine) }\end{array}$ \\
\hline Venetoclax (November 2018) & $\mathrm{BCL}-2$ inhibitor & $\begin{array}{l}\text { In combination with azacitidine or decitabine, or low-dose cytarabine } \\
\text { in newly diagnosed AML aged } \geq 75 \text { years or with co-morbidities that } \\
\text { preclude the use of intensive induction chemotherapy }\end{array}$ \\
\hline Enasidenib (August 2017) & IDH2 inhibitor & $\begin{array}{l}\text { Relapsed or refractory IDH2- mutated AML (as detected by FDA- } \\
\text { approved test) }\end{array}$ \\
\hline Ivosidenib (July 2018) (May 2019) & IDH1 inhibitor & $\begin{array}{l}\text { 1. Relapsed or refractory IDH1-mutated (susceptible mutation, as } \\
\text { detected by FDA-approved test) AML. 2. First line treatment of IDH1- } \\
\text { mutated AML (as detected by FDA-approved test), patients } \geq 75 \text { years } \\
\text { old or ineligible to receive intensive chemotherapy. }\end{array}$ \\
\hline Gilteritinib (November 2018) & FLT3 inhibitor & $\begin{array}{l}\text { Patients with relapsed or refractory FLT3-mutated AML (as detected by } \\
\text { FDA-approved test) }\end{array}$ \\
\hline CC-486 (September 2020) & $\begin{array}{l}\text { Oral azacitidine hypomethylating agent } \\
\text { (30\% absorption) approved at } 300 \mathrm{mg} \\
\text { daily } \times 14 \text { every month }\end{array}$ & $\begin{array}{l}\text { Continued treatment of adult patients with } \mathrm{AML} \text { who achieved first } \\
\text { complete remission or complete remission with incomplete blood } \\
\text { count recovery following intensive induction chemotherapy and who } \\
\text { are not able to complete intensive curative therapy }\end{array}$ \\
\hline $\begin{array}{l}\text { Oral Decitabine-cedazuridine } \\
\text { (July 2020) }\end{array}$ & $\begin{array}{l}\text { Oral hypomethylating agent }(100 \% \\
\text { absorption) }\end{array}$ & $\begin{array}{l}\text { Alternative to parenteral HMAs decitabine for the treatment of adults } \\
\text { with MDS (pretreated/untreated; de novo/secondary) or CMML }\end{array}$ \\
\hline
\end{tabular}

16(p13; q22)] or $\mathrm{t}(16 ; 16)(\mathrm{p} 13 ; \mathrm{q} 22)$ and $\mathrm{t}(8 ; 21)(\mathrm{q} 22 ; \mathrm{q} 22$,$) ,$ together referred to as CBF AML; diploid karyotype (about $40-50 \%$ of patients); complex karyotype (three or more chromosomal abnormalities); others.

Molecular subsets also define prognosis and are therapeutically targetable. Among patients with a diploid karyotype, single mutations and mutation combinations interact differently, in sometimes intricate balancing acts. For example, a mutation of nucleophosmin-1 (NPM1) without a FLT3 mutation is associated with a more favorable outcome. If a FLT3 mutation, particularly FLT3 internal tandem duplication (FLT3-ITD), is present (about $50 \%$ of patients with a diploid karyotype and NPM1 mutation), then the outcome was worse historically, and largely dependent on the FLT3 allelic ratio (AR). In newly diagnosed FLT3-mutated AML, the AR of FLT3-ITD to FLT3-wild type strongly influenced outcome in several studies of chemotherapy-based therapies that did not include FLT3 inhibitors ${ }^{34-36}$. The FLT3-ITD AR is defined as the ratio of the area under the curve of "FLT3ITD" divided by the area under the curve of "FLT3wildtype" using a semi-quantitative DNA fragment analysis $^{30}$. A higher FLT3-ITD AR (generally defined as $\geqslant 0.5$ ) is associated with worse survival than lower ratios, likely reflecting increased FLT3 dependency in cases with high ARs. This may change with the incorporation of FLT3 inhibitors into AML chemotherapy and into post stem cell transplantation (SCT) maintenance. Mutations, including $A S X L 1, R U N X 1, T P 53$, and others may also associate with outcome differences. Several molecular mutations are potentially targetable (Table 3$)^{30-51}$.

Next-generation sequencing identified multiple recurrent somatic mutations in $>90 \%$ of patients with $\mathrm{AML}^{21,52}$. Frequently mutated genes (frequency $>5 \%$ ) are FLT3, NPM1, DNMT3A, IDH1, IDH2, TET2, RUNX1, p53, NRAS, CEBPA, WT1 $1^{21,24,52}$. Based on functional analysis and known pathways, these are routinely grouped into biologic- functional categories: myeloid 
Table 2 Cytogenetic-molecular entities in acute myeloid leukemia (NCCN classification).

\begin{tabular}{|c|c|c|}
\hline NCCN & Cytogenetics & Molecular abnormalities \\
\hline Better risk & $\begin{array}{l}\text {-Inversion (16) or translocation }(16 ; 16) \\
\text {-Translocation }(8 ; 21) \\
\text {-Translocation }(15 ; 17)\end{array}$ & $\begin{array}{l}\text { Normal cytogenetics: NPM1 mutation in the absence of FLT3-ITD or FLT3-ITD low } \\
\text { allelic ratio; isolated biallelic CEBPA mutation }\end{array}$ \\
\hline Intermediate risk & $\begin{array}{l}\text {-Normal cytogenetics } \\
\text {-Trisomy } 8 \text { alone } \\
\text {-Translocation }(9 ; 11) \\
\text {-Other non-defined }\end{array}$ & $\begin{array}{l}\text { - Translocation (8;21), inversion (16), translocation (16;16): with c-KIT mutation } \\
\text { - NPM1-mutated and FLT3-ITD mutated (high allelic ratio) } \\
\text { - NPM1-wild type and FLT3- wild type } \\
\text { - NPM1-wild type and FLT3-ITD mutated (low allelic ratio) }\end{array}$ \\
\hline Poor risk & $\begin{array}{l}\text {-Complex ( } \geq 3 \text { clonal chromosomal } \\
\text { abnormalities) } \\
\text {-Monosomal karyotype: }-5,5 q-, 7,7 q- \\
\text {-11q23-non translocation }(9: 11) \\
\text {-Inversion (3), translocations of }(3 ; 3) \\
\text {-Translocation (6;9) or (9;22) }\end{array}$ & $\begin{array}{l}\text { - TP53 mutation } \\
\text { - RUNX1 mutation } \\
\text { - ASXL1 mutation } \\
\text { - NPM1-wildtype and FLT3-ITD mutated (high allelic ratio) }\end{array}$ \\
\hline
\end{tabular}

Notes related to the NCCN Risk classification:

(1) The NCCN classification is applicable to younger patients with AML (age up to 60-65 years old) and in de novo AML. Older patients with AML and patients with secondary (progression to AML from myelodysplastic syndrome, particularly if treated; or from myeloproliferative neoplasm) or therapy-related AML have significantly poorer outcome within each of the NCCN risk categories (the exception being possibly APL).

(2) At MD Anderson, all translocations involving 11q23 are considered adverse. Also, in updated analyses, a translocation (9;11) may be intermediate risk only in de novo younger AML (but not in older or secondary/therapy-related AML).

(3) The differential effect of mutations is particularly notable in patients with diploid or intermediate risk karyotype, but not in patients with better or poor risk karyotypes.

Low allelic ratio is $<0.5$; high allelic ratio is $\geq 0.5$.

Adapted from National Cancer Centers Network (NCCN). Accessed October 9, 2020. https://www.nccn.org/

NCCN National Cancer Centers Network.

transcription-factor fusions or mutations; NPM1 mutations; tumor-suppressor gene mutations; epigenomemodifying gene mutations; activated signaling-pathway gene mutations; cohesin-complex gene mutations; and spliceosome-complex gene mutations. These mutations exhibit shared co-occurrences or exclusive dissociations that help identify AML pathways of clonal dominance and shifts that would result into more rational targeting therapies.

Translating to clinical practice, the important molecular subsets are based on the identification of a FLT3 mutation (30\% of AML), NPM1 mutation (40-50\% of normal karyotype AML), isocitrate dehydrogenase 1 or $2($ IDH1/2) mutations (20\% of AML), and TP53 mutations (2 to $20 \%$ of AML).

Patients with NPM1-mutated AML have a more favorable prognosis; those with FLT3-ITD mutations have a poor prognosis, especially among patients with high FLT3 ARs and in the absence of NPM1 mutation. Patients with diploid karyotype AML (without adverse mutations such as TP53, or ASXL1) and biallelic CEBPA mutations (2\% or less of AML) have a favorable prognosis 5 .

The FLT3 mutations, including FLT3-ITD and FLT3tyrosine kinase domain (TKD) point mutations (D835 most common), can now be targeted with FLT3 inhibitors. Midostaurin and gilteritinib are type I FLT3 inhibitors and suppress both FLT3-ITD and FLT3-TKD mutations. Sorafenib and quizartinib are type II FLT3 inhibitors that target only FLT3-ITD.

The IDH1/2 mutations can be targeted with novel IDH inhibitors, ivosidenib, which targets IDH1 mutations, and enasidenib, which targets $I D H 2$ mutations. The $I D H 1 / 2$ mutations also generate AML dependence on BCL-2 for survival, rendering them responsive to venetoclax-based therapy $^{53}$.

Mutations of epigenetically related molecular events (DNMT3A, IDH1/2, TET2, ASXL1, and MLL1) may suggest the possible benefit of epigenetic-targeted therapy.

In CBF AML, mutations in $c-K I T$ may be associated with worse outcome in some studies ${ }^{47-50}$, but not with fludarabine-cytarabine-GO-based regimens ${ }^{12-14}$. Investigating the addition of a potent $\mathrm{C}-\mathrm{KIT}$ inhibitor (avapritinib, dasatinib) to chemotherapy in $c$-KIT-mutated CBF AML is of interest ${ }^{50,51}$.

Mutations and/or deletions of the tumor-suppressor gene TP53 (located on the short arm of chromosome 17) occur in $2-20 \%$, are more common in older patients and patients with secondary or therapy-related AML, and are associated with complex cytogenetics ${ }^{38-41}$. In a study of 293 patients, 53 (18\%) had TP53 mutations; these were associated with complex karyotype $(p<0.001)$ and with abnormalities of chromosomes 17 and 5 and/or 7, and with a low $C R$ rate and short survival ${ }^{39}$. Most patients with TP53 mutations may not benefit from intensive 
Table 3 Clinically relevant mutations in acute myeloid leukemia.

\begin{tabular}{|c|c|c|}
\hline Mutation & $\begin{array}{l}\% \text { Incidence (with diploid } \\
\text { karyotype) }\end{array}$ & Comments \\
\hline FLT3-ITD & $20(30-35)$ & $\begin{array}{l}\text { Adverse prognosis - high allelic ratio is an indication for allogeneic SCT; adding FLT3 inhibitors as post } \\
\text { SCT maintenance. } \\
\text { Outcome may change with the addition of FLT3 inhibitors to chemotherapy (sorafenib, midostaurin, } \\
\text { gilteritinib) }\end{array}$ \\
\hline FLT3-TKD & $5-10$ & Prognostic significance uncertain; response to Type I FLT3 inhibitors like gilteritinib and midostaurin. \\
\hline NPM1 & $30(40-50)$ & $\begin{array}{l}\text { FLT3 wild-type NPM1-mutated = favorable prognosis. Older patient }+ \text { NPM1-mutated AML }=\text { more } \\
\text { sensitive to cytarabine and hypomethylating agents }+ \text { venetoclax }\end{array}$ \\
\hline CEBPA & $<5$ & Biallelic mutations $=$ better prognosis (without concomitant unfavorable mutations) \\
\hline DNMT3A & $20(30-35)$ & $\begin{array}{l}\text { Associated with NPM1 and FLT3-ITD. } \\
\text { Adverse prognosis, especially with concomitant FLT3 mutations; epigenetic modulation }\end{array}$ \\
\hline RUNX1 & 10 & Adverse prognosis \\
\hline ASXL1 & $10-15$ & Adverse prognosis \\
\hline KIT & 5 & $\begin{array}{l}\text { Incidence higher in CBF-AML;? unfavorable outcome in CBF-AML (? need for c-KIT inhibitors). Possible } \\
\text { benefit from addition of gemtuzumab ozogamicin }\end{array}$ \\
\hline NRAS & $10-15$ & $\begin{array}{l}\text { 40-50\% of inversion } 16 \text { AML; no definite prognostic association; may be a mechanism of resistance to } \\
\mathrm{BCL} 2, \mathrm{IDH} \text {, and FLT3 inhibitors (especially Type I) at the time of relapse. }\end{array}$ \\
\hline IDH2 & $10-20(20-30)$ & Therapy with enasidenib and/or venetoclax-based combinations \\
\hline IDH1 & $7-10(10-15)$ & Therapy with ivosidenib and/or venetoclax-based combinations \\
\hline TET2 & $10-15$ & Adverse prognosis; epigenetic modulation \\
\hline TP53 & $2-20$ & $\begin{array}{l}\text { High incidence (70\%) in complex karyotype; very adverse prognosis. Limited benefit of intensive } \\
\text { chemotherapy among those with allelic frequency of } \geq 40 \% \text {. Investigational approaches should be } \\
\text { considered (i.e., APR-246; magrolimab and other anti-CD47 antibodies) }\end{array}$ \\
\hline
\end{tabular}

Prognostic impact of mutations mostly in the context of normal karyotype.

chemotherapy and may have similar or improved outcomes and less toxicity with lower intensity approaches $^{40,54}$. The variant allelic frequency (VAF; percent mutated/total) of TP53 mutations may help select patients who would not benefit from intensive induction therapy. Novel strategies like APR-246 or magrolimab have shown promise.

Patients with the cytogenetic-molecular subset of "mixed-lineage leukemia" (translocations involving 11q23; $M L L 1, K M T 2 A$ rearrangement) may respond well to the novel menin inhibitors (SNDX-5613, KO-539, others) ${ }^{55}$.

Translocations involving chromosome 3q26.2 (EVI1), location of the MECOM (MDS1 and EVI1 complex locus) gene, have an extremely poor outcome with standard chemotherapy ${ }^{46}$. Additional mutations associated with adverse outcomes are DNMT3A $A^{42,43}, A S X L 1$, RUNX $1^{44,45}$, and others ${ }^{40-44}$.

\section{Measurable residual disease in complete remission}

Measuring residual disease in AML in complete remission (CR) is now part of the standard of care in
$\mathrm{AML}^{56-62}$. The detection of measurable residual disease (MRD) at the time of morphologic CR is associated with a higher relapse rate and with worse survival in AML. Measurable residual disease has been commonly investigated using two methodologies, multi-color flow-cytometric measurements of MRD (MFC-MRD), and molecular quantification of residual disease ${ }^{56-61}$.

Polymerase chain reaction (PCR) measure of residual molecular disease is routinely used to monitor quantitatively unique AML-defining translocations and mutations in APL, CBF AML, NPM1-mutated AML, and now expanding to other molecular subsets (IDH1/2 and FLT3 mutations). In APL, PCR quantification of promyelocytic leukemia-retinoic receptor alpha (PML-RAR alpha) may detect early molecular relapse ${ }^{63}$. The same is true for CBF AML. Inversion 16 and $t(16 ; 16)$ result in the formation of the CBF beta/myosin heavy chain $11(C B F B / M Y H 11)$ fusion gene. The $t(8 ; 21)$ leads to the formation of the Runt-related transcription factor 1 [RUNX1]/RUNX1T1 (RUNX1/RUNX1T1) fusion gene. Detection of molecular fusion genes MRD by quantitative PCR in CBF AML (especially AML with inversion 16) predicts for 
relapse $^{64,65}$. Interestingly, patients with $\mathrm{t}(8 ; 21)$ may have persistent MRD at levels below $0.1 \%$, but still remain in durable complete remissions and possibly cured. Among patients with non-CBF non-APL AML, monitoring mutations by next-generation sequencing is informative when possible, for example in patients with NPM1 mutations $^{66,67}$. Combining MFC and molecular PCR studies may improve on the capability of MRD studies to predict for relapse ${ }^{56}$. Better outcomes are reported in FLT3-mutated and IDH-mutated AML with molecular MRD clearance.

Measurable residual disease in $\mathrm{CR}$ indicates worse prognosis due to a higher risk of relapse. This should lead to consideration of therapeutic interventions. In APL, therapy at the time of molecular relapse improved survival compared with therapy at the time of hematologic relapse $^{63}$. Allogeneic SCT for persistent MRD in CR in CBF AML improved survival compared with continuation of standard therapy ${ }^{65}$. Important interventions in AML with MRD in CR may include allogeneic SCT; investigational approaches with more intensified chemotherapy regimens, or with HMAs (parenteral or newly approved oral formulations) plus venetoclax; targeted therapy combinations when indicated for particular molecular abnormalities (FLT3 or IDH inhibitors); antibody therapies (e.g., CD123 or CD33 monoclonal or BiTEs); or immune therapies (e.g., checkpoint inhibitors). However, the persistence of DTA mutations in CR (mutations in DNMT3A, TET2, ASXL1) does not predict for relapse ${ }^{56}$.

\section{Treatment of AML}

The heterogeneous group of AML disorders requires different selective therapies. Next, we will discuss the treatment of the highly curable leukemias, APL and CBF AML; the different therapeutic approaches in younger and older patients with AML; and the addition of the novel targeted therapies (venetoclax, FLT3 inhibitors, and IDH inhibitors) to standard therapies.

\section{Acute promyelocytic leukemia}

Acute promyelocytic leukemia represents $5-10 \%$ of AML and is defined by the cytogenetic abnormality $\mathrm{t}(15$; 17), which results in the PML-RAR alpha fusion oncogene and its encoded oncoprotein. The PML-RAR $\alpha$ oncoprotein acts as a dominant negative inhibitor of wild-type RAR $\alpha$, causing a maturation block and the clinicalpathologic picture of APL.

Combinations of anthracyclines and cytarabine first established the potential cure rate of $30-40 \%$ in $\mathrm{APL}^{68,69}$. The early mortality from disseminated intravascular coagulopathy (DIC) and bleeding with anthracyclinescytarabine was significant, about $10-20 \%$. The added antiAPL efficacy of high-dose cytarabine and maintenance chemotherapy (POMP) was modest at best ${ }^{69}$.
In the late 1980s and early 1990s, the major anti-APL efficacies of ATRA and arsenic trioxide were discovered. Gemtuzumab ozogamicin was also highly effective in $\mathrm{APL}^{70}$. The most potent anti-APL drugs are arsenic trioxide, followed by ATRA, GO, and anthracyclines.

Based on the single-agent anti-APL efficacies of ATRA and arsenic trioxide ${ }^{71}$, ATRA was initially added to chemotherapy during both induction and/or consolidation ${ }^{72-74}$, and arsenic trioxide was investigated initially in APL relapse and later as consolidation therapy ${ }^{75}$. Comparative studies showed that the addition of ATRA to chemotherapy during induction and/or consolidation improved survival ${ }^{71-73}$, and that the addition of arsenic trioxide during consolidation in $\mathrm{CR}$ also improved event-free survival (EFS). In the late 1990's, the combination of idarubicin (or other anthracyclines) and ATRA (AIDA regimen) became standard of care in $\mathrm{APL}^{76}$.

\section{Chemotherapy-free regimens: ATRA and arsenic trioxide}

The MD Anderson group first investigated the use of non-chemotherapy regimens including ATRA, arsenic trioxide and GO, and demonstrated the high efficacy of this strategy ${ }^{8}$. Randomized studies confirmed the superiority of ATRA plus arsenic trioxide over AIDA in low and intermediate risk $\mathrm{APL}^{11,77}$. A SWOG study also demonstrated the efficacy and safety of ATRA with arsenic trioxide and $\mathrm{GO}$ in high risk $\mathrm{APL}^{78}$. With the ATRA plus arsenic trioxide regimens, the CR rate is $90+\%$ and the cure rates $80+\%$. Induction mortality from DIC is low (about $5 \%$ ). Resistant disease is extremely rare, except in molecular variant-APL (translocations between chromosome 11 and 17 [PLZF-RAR alpha], or between chromosome 5 and 17).

Important considerations in APL management are: (1) Granulocyte-colony stimulating growth factors (filgrastim, pegfilgrastim) should never be used in APL, as it is the one leukemia where granulocyte growth factors may induce a drastic increase in APL progression, and trigger fatal $\mathrm{DIC}^{79}$. (2) Watch for fluid overload (often confused with "differentiation syndrome"). This is related to ATRA and arsenic trioxide, as well as the use of high-volume blood product transfusions (fresh frozen plasma) to prevent the complication of consumptive coagulopathy. These complications are best managed by holding ATRA-arsenic trioxide therapy briefly and with aggressive diuresis ${ }^{80}$. (3) The development of a "differentiation syndrome" with possible multiorgan failure; this requires the use of prophylactic steroids during induction (together with antibiotics and antifungal prophylaxis). (4) Among patients with CNS bleeding at diagnosis, the risk of CNS leukemia may increase; two intrathecal cytarabine injections in CR may eliminate this rare complication.

The MRC comparative trial investigated a lower dose schedule of arsenic trioxide $0.3 \mathrm{mg} / \mathrm{kg}$ on Days $1-5$ during 


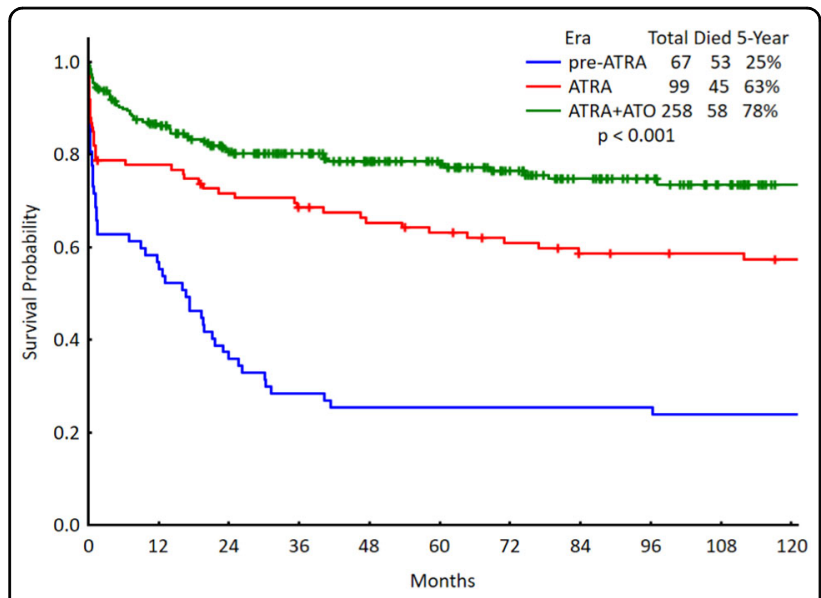

Fig. 2 Survival of acute promyelocytic leukemia at MD Anderson (1970-2020).

week 1 , then $0.25 \mathrm{mg} / \mathrm{kg}$ twice weekly in weeks $2-8$ of Course 1 followed by 4 consolidations courses (63 arsenic trioxide doses) ${ }^{77}$.

Oral formulations of arsenic trioxide are under investigation; these would render the treatment of APL more convenient, particularly during the longer term consolidation $^{81,82}$

Figure 2 shows the MD Anderson results in APL, and the significant outcome improvement in the era of ATRA and arsenic trioxide.

\section{Core-binding factor acute myeloid leukemia}

The CBF AMLs include the subsets with chromosomal abnormalities involving inversion $16 / \mathrm{t}(16 ; 16)$ or $\mathrm{t}(8 ; 21)$. These constitute $10-15 \%$ of adult AML cases.

The use of established chemotherapy drugs in optimized combinations has gradually improved the cure rates in CBF AML from $<50 \%$ to about $75 \%{ }^{13-17}$. Historically, CBF AML was treated with cytarabine plus anthracycline induction chemotherapy followed by 1-4 high-dose cytarabine consolidations. The cure rates were $30-40 \%$ with one consolidation versus $50+\%$ with $3-4$ consolidations $^{83,84}$. Using induction- consolidation courses of high-dose cytarabine combinations with fludarabine and idarubicin, and the addition of GO $3 \mathrm{mg} / \mathrm{m}^{2} \times 1$ during induction and consolidation (comparative SWOG and MRC studies) resulted in estimated 5-year survival rates of $75+\%$ in $\mathrm{CBF} \mathrm{AML}^{13-17}$. At MD Anderson, we currently use fludarabine, high-dose cytarabine and GO (i.e., FLAG-GO) during induction and consolidations, for a total of up to six courses, and modify therapy with the addition of maintenance for persistent MRD at the completion of therapy. The results were better when GO replaced idarubicin. The 5-year survival rates were $80 \%$ in both inversion 16 and $t(8 ; 21)$ AML (Fig. 3) $)^{3,14}$. The MRC trials using the fludarabine, high-dose cytarabine and

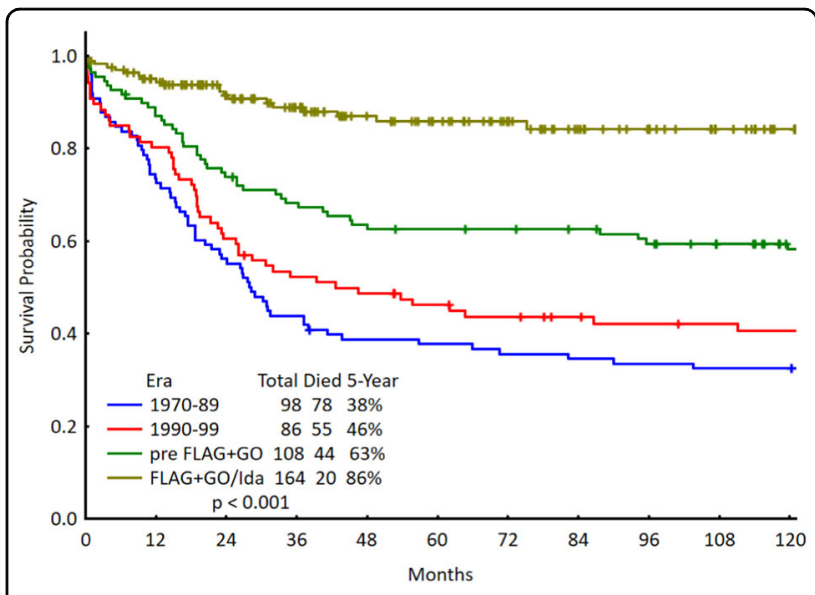

Fig. 3 Survival of core-binding factor acute myeloid leukemia at MD Anderson (1970-2020).

idarubicin combination (FLAG- IDA regimen) $+/-$ GO also reported cure rates of $80+\%$ in $\mathrm{CBF}_{\mathrm{AML}}{ }^{15}$. In a metaanalysis of five studies, adding GO to standard inductionconsolidation therapy improved survival from 50 to $75 \%{ }^{17}$.

Today, GO should always be added to the standard chemotherapy in CBFAML. The new regimens utilizing fludarabine, high-dose cytarabine and GO, with or without idarubicin, may be better, producing cure rates of $75-80+\%{ }^{14,15}$.

The CBF AML often exhibits co-occurrence of mutations in FLT3 (15-20\%), c-KIT (29-30\%), RAS (30-50\%), and others. Some studies report $c-K I T$ or multiple mutations to be associated with worse prognosis ${ }^{47-49}$. This has not been our experience with the FLAG-GO/ idarubicin regimen where the efficacy of the regimen may have overcome the adverse effects of these mutations. Recent studies suggest an adverse impact of epigenetic mutations (ASXL2 or cohesin/spliceosome mutations). Older patients with CBF AML are treated with lower adjusted dose FLAG-GO/IDA. Patients who cannot tolerate FLAG-GO/IDA or who have persistence molecular MRD positivity may be offered HMA therapy (decitabine, azacitidine) with venetoclax/GO, the treatment duration adjusted according to the MRD results or for $12+$ months. Targeted therapies may also be considered (avapritinib or dasatinib for c-KIT mutations; FLT3 inhibitors for FLT3 mutations) ${ }^{50,51}$.

Figure 3 shows the MD Anderson outcomes in CBF AML over the decades.

\section{Younger patients with acute myeloid leukemia (and/or older patients fit for intensive chemotherapy)}

The median age in AML is 68 years ${ }^{85}$. Most of the research with $3+7$ and other intensive chemotherapy regimens was conducted in younger patients (usual upper 
age limit $60-65$ years). The published results of these trials may not reflect the actual results in the community practice (discussed later) ${ }^{85}$.

\section{The " $3+7$ " anthracyclines-cytarabine regimens; high-dose cytarabine consolidations}

The discovery of the anti-AML activity of cytarabine and anthracyclines in the 1970s led to a series of randomized trials evaluating different doses and schedules of cytarabine (5 versus 7 versus 10 days; $100 \mathrm{mg} / \mathrm{m}^{2}$ versus $200 \mathrm{mg} / \mathrm{m}^{2}$ ) in combination with anthracyclines, and the addition of other agents (etoposide, 6-mercaptopurine, 6thioguanine, others) to induction-maintenance therapy. These studies established the $3+7$ regimen as a standard of care over the next 40 years. The $3+7$ refers to 3 days of anthracyclines (daunorubicin $30-60 \mathrm{mg} / \mathrm{m}^{2}$ intravenously [IV] daily $\times 3$; idarubicin $12 \mathrm{mg} / \mathrm{m}^{2}$ IV daily $\times 3$ days) and cytarabine $\left(100-200 \mathrm{mg} / \mathrm{m}^{2}\right.$ IV as a continuous infusion daily for 7 days). Consolidation strategies have investigated multiple courses of chemotherapy with cytarabine and anthracyclines, as well as high-dose cytarabine. A randomized trial by Meyer and the Cancer and Acute Leukemia Group B (CALGB) reported significantly superior survival using high-dose cytarabine consolidation therapy ( $3 \mathrm{~g} / \mathrm{m}^{2}$ IV over $2-3 \mathrm{~h}$ every $12 \mathrm{~h}$ on Days 1,3 , and 5) for four courses, compared with lower cytarabine dose schedules $^{86}$. In the CALGB study, high-dose cytarabine consolidations were followed by four additional courses of $2+5$ chemotherapy. The latter addition was omitted from the subsequent comparative trials, which may be important (later studies using this regimen reported 5year survival rates of $20-30 \%$ rather than $40 \%)^{7}$. Highdose cytarabine then became the consolidation standard of care in AML. Other studies investigated lower doses of high-dose cytarabine $\left(1.5 \mathrm{~g} / \mathrm{m}^{2}\right), 4-5$ courses versus lower numbers of consolidation courses, and the possible benefits of using allogeneic or autologous SCT in first $\mathrm{CR}^{87}$.

\section{Better regimens than $3+7$}

An increasing body of research suggests that there are better induction-consolidation regimens than $3+7$. Modifications of frontline AML therapy include: (1) The use high-dose cytarabine combination during induction. (2) Optimization of the dose of daunorubicin $\left(60 \mathrm{mg} / \mathrm{m}^{2}\right.$ daily $\times 3$, versus $45 \mathrm{mg} / \mathrm{m}^{2}$ or $90 \mathrm{mg} / \mathrm{m}^{2}$ daily $\times 3$ ) and the use of other anthracyclines (idarubicin, mitoxantrone). (3) The addition of adenosine nucleoside analogs (fludarabine, clofarabine, cladribine) to cytarabine-anthracyclines. (4) The addition of the CD33-targeted monoclonal antibody (GO). (5) The addition of targeted therapies such as FLT3 and IDH inhibitors in appropriate patients. (6) The addition of the BCL-2 inhibitor venetoclax to induction therapy on investigational trials. (7) The use of maintenance therapy with oral azacitidine.

\section{High-dose cytarabine induction}

High-dose cytarabine (1-3 g/m $\mathrm{m}^{2}$ twice daily on Days 1,3 and 5 or daily $\times 5$ ) in AML consolidation is an established standard of care ${ }^{86,88,89}$. Several studies evaluated highdose cytarabine during induction. A SWOG trial randomizing younger patients ( $<65$ years) to standard-dose cytarabine $\left(200 \mathrm{mg} / \mathrm{m}^{2}\right.$ daily $\left.\times 7\right)$ versus high-dose cytarabine $\left(2 \mathrm{~g} / \mathrm{m}^{2}\right.$ every $\left.12 \mathrm{~h} \times 12\right)$ during induction (both with daunorubicin) showed a higher 4-year relapse-free survival (RFS) rate with high-dose cytarabine among younger ( $<50$ years; $33 \%$ versus $21 \%$ ) and older patients (50 to 64 years; $21 \%$ versus $9 \% ; p=0.049)^{88}$. An Australian study randomizing 301 younger patients (60 years or less) to high-dose cytarabine $\left(3 \mathrm{~g} / \mathrm{m}^{2}\right.$ every $\left.12 \mathrm{~h} \times 8\right)$ or standarddose cytarabine (both with daunorubicin and etoposide induction) reported significant improvements in $C R$ duration (median 45 versus 12 months; $p=0.0004$ ) and 5year RFS rate $(49 \%$ versus $24 \%)$ with high-dose cytarabine $^{89}$. A meta-analysis of three trials in 1691 patients randomized to induction therapy with high-dose versus standard- dose cytarabine reported improved 4-year rates of RFS ( $p=0.03)$, overall survival $(p=0.0005)$ and EFS $(p$ $<0.0001)$ with high-dose cytarabine ${ }^{90}$.

Lowenberg and colleagues ${ }^{91}$ randomized 858 younger patients (median age 49 years; range 18 to 60 years) to induction therapy with high-dose cytarabine $1 \mathrm{~g} / \mathrm{m}^{2}$ every $12 \mathrm{~h} \times 10$ versus standard-dose cytarabine $200 \mathrm{mg} / \mathrm{m}^{2}$ daily $\times 7$, both in combination with idarubicin. They reported similar CR, EFS, and survival rates in the two study arms. This study results may have been confounded by the study design, in which all patients received highdose cytarabine during induction Course 2 (either $2 \mathrm{~g} / \mathrm{m}^{2}$ every $12 \mathrm{~h} \times 8$-total dose $16 \mathrm{~g} / \mathrm{m}^{2}$-for patients randomized to high-dose cytarabine during Course 1 ; or cytarabine $1 \mathrm{~g} / \mathrm{m}^{2}$ every $12 \mathrm{~h} \times 6$ days-total dose $12 \mathrm{~g} /$ $\mathrm{m}^{2}$-for patients randomized to standard-dose cytarabine during Course 1). Thus, all patients received high- dose cytarabine during the two induction courses.

Willemze and colleagues ${ }^{92}$ (EORTC-GIMEMA) conducted a randomized trial in which 1942 younger patients (60 years or less) received daunorubicin plus etoposide and high-dose cytarabine $3 \mathrm{~g} / \mathrm{m}^{2}$ every $12 \mathrm{~h} \times 8$ versus standard-dose cytarabine $100 \mathrm{mg} / \mathrm{m}^{2}$ daily $\times 10$. Highdose cytarabine was associated with significantly higher CR rates $(82 \%$ versus $76 \% ; p=0.01)$, 6-year EFS rates ( $44 \%$ versus $35 \% ; p=0.003$ ), and 6 -year survival rates (52\% versus $43 \% ; p=0.009$ ) among patients $15-45$ years old. Among patients 45-60 years, high-dose cytarabine was also associated with significant improvements in CR and 6-year EFS rates, as well as a trend for better survival among patients with FLT3-ITD AML or poor prognosis karyotypes.

Bassan and colleagues ${ }^{93}$ randomized 574 patients (median age 52 years; range 16 to 73 years) to ICE (idarubicin- 
cytarabine-etoposide) or idarubicin plus sequential highdose cytarabine (2-weekly 3-day blocks of cytarabine $2 \mathrm{~g} / \mathrm{m}^{2}$ twice daily $\times 2$ days). Sequential high-dose cytarabine induction was associated with a significantly higher $\mathrm{CR}$ rate post Course $1(81 \%$ versus $69 \% ; p=0.02)$, and significantly better rates of 5 -year survival ( $49 \%$ versus $39 \%$; $p=0.045$ ) and RFS ( $48 \%$ versus $36 \% ; p=0.028$ ).

A recent SWOG trial (SWOG-1203) randomized patients to: (1) $3+7$ induction followed by four consolidations with high-dose cytarabine $\left(3 \mathrm{~g} / \mathrm{m}^{2}\right.$ twice daily on Days 1,3 , and 5-total cytarabine $18 \mathrm{~g} / \mathrm{m}^{2} /$ course x $4=72 \mathrm{~g} / \mathrm{m}^{2}$ ), (2) IA regimen:Idarubicin plus continuous high-dose cytarabine $\left(1.5 \mathrm{~g} / \mathrm{m}^{2}\right.$ continuous infusion daily $\left.\times 4\right)$ followed by IA consolidations with cytarabine $0.75 \mathrm{~g} / \mathrm{m}^{2}$ continuous infusion daily $\times 3$ days $\left(=2.25 \mathrm{~g} / \mathrm{m}^{2} /\right.$ course $) \times 4$ (total cytarabine $\left.15 \mathrm{~g} / \mathrm{m}^{2}\right)$; (3) IA + vorinostat ${ }^{94}$. While the latter two arms were presumably testing the benefit of high-dose cytarabine induction, the total dose of cytarabine was 4.5 times higher with the $3+7$ regimen compared with the IA regimen. As expected, the $3+7$ regimen, delivering more total high-dose cytarabine, was superior in the CBF AML. However, despite the lower total cytarabine dose given in IA, the results of $3+$ 7 and IA were similar among patients with intermediate or adverse karyotypes. The design of this trial unfortunately did not allow a real testing of the benefit of high-dose cytarabine added to induction.

\section{Addition of nucleoside analogs}

A combination regimen of fludarabine, high-dose cytarabine and idarubicin combination (FLAG-IDA or FAI), was developed at MD Anderson based the preclinical studies of Plunkett et al. ${ }^{95-98}$. The Medical research Council (MRC) AML 15 randomized trial compared the FLAG-IDA in younger patients with AML to $3+7$ regimens without or with etoposide. The FLAGIDA regimen consists of cytarabine $2 \mathrm{~g} / \mathrm{m}^{2}$ daily for 5 days, fludarabine $30 \mathrm{mg} / \mathrm{m}^{2}$ daily for 5 days, and idarubicin $8-10 \mathrm{mg} / \mathrm{m}^{2}$ daily for 3 days. Among patients who tolerated four courses on the FLAG-IDA arm (2 FLAGIDA +2 high-dose cytarabine), the 8 -year survival rate was $66 \%$ versus $47 \%$ in the standard arm ${ }^{15,87,98}$. The FLAG-IDA/FAI is intensive and difficult to deliver due to side effects related to myelosuppression, but likely not more than allogeneic SCT, and possibly worth a $20 \%$ difference in 8-year survival. The FLAG-IDA/FAI is not a simple exploration of high-dose cytarabine, but a multifaceted strategy (addition of fludarabine, idarubicin instead of daunorubicin, high-dose cytarabine induction) that may be better than $3+7$ when administered at AML centers of excellence. Improved leukemia management expertise (supportive care; antibiotics and antifungal prophylaxis; timely transfusions support, management of toxicity and treatment of infections/sepsis) would allow safe and full delivery of this regimen.
The optimal dose of high-dose cytarabine is unknown even after $30+$ years of research of different high-dose cytarabine schedules. Cytarabine $3 \mathrm{~g} / \mathrm{m}^{2}$ may be beyond the dose required to maximize the anti-AML effect, and may increase toxicity. High-dose cytarabine $1.5-2 \mathrm{~g} / \mathrm{m}^{2}$ may be equally effective and less toxic. The MRC studies compared high-dose cytarabine $1.5 \mathrm{~g} / \mathrm{m}^{2}$ versus $3 \mathrm{~g} / \mathrm{m}^{2}$ during consolidation, and four versus five courses, reporting equivalent results $^{87}$. A study from Korea showed that high-dose cytarabine $1.5 \mathrm{~g} / \mathrm{m}^{2}$ or more during consolidation was associated with better RFS and survival rates compared with cytarabine $1 \mathrm{~g} / \mathrm{m}^{299}$. At MD Anderson, we use high-dose cytarabine $1.5-2 \mathrm{~g} / \mathrm{m}^{2}$ daily $\times 5$ (total $7.5-10 \mathrm{~g} / \mathrm{m}^{2}$ per course) during induction and consolidations.

Other adenosine nucleoside analogs (clofarabine, cladribine) have also been explored in combinations with standard chemotherapy.

The Polish investigators added cladribine to frontline $3+7$ induction chemotherapy in two sequential randomized trials. In the first study, they randomized 400 patients to induction with $3+7+/-$ cladribine, and reported that adding cladribine produced higher CR $(64 \%$ versus $46 \% ; p=0.0009$ ) and leukemia-free survival rates $(44 \% \text { versus } 28 \% ; p=0.05)^{100}$. In the subsequent study, they compared three arms, two of them adding cladribine or fludarabine. They showed again that the addition of cladribine (but not fludarabine) resulted in higher CR $(67.5 \%$ versus $56 \% ; p=0.001)$ and 3 -year survival rates $(45 \% \text { versus } 33 \% ; p=0.02)^{101}$.

At MD Anderson, we continue to use AML regimens that add adenosine nucleoside analogs like fludarabine (FAI, FLAG-IDA regimens), clofarabine (CIA regimen) and cladribine (CLIA regimen) to idarubicin and highdose cytarabine as frontline induction therapy in younger patients with $\mathrm{AML}^{102}$. All patients with FLT3-mutated AML now receive gilteritinib or quizartinib during induction and consolidation. Based on the positive experiences of combining FLT3 inhibitors (midostaurin, sorafenib, gilteritinib) with chemotherapy from pilot studies and from the German and Intergroup randomized trials (discussed later), this approach may become a standard of care in FLT3- mutated AML, but also perhaps in all patients with AML regardless of FLT3 mutation status.

\section{Choice of anthracycline}

The better anthracycline and its optimal dose have been the subject of several randomized trials. Historically, daunorubicin $30-60 \mathrm{mg} / \mathrm{m}^{2}$ daily $\times 3$ was used for induction therapy. Two studies compared higher-dose daunorubicin $90 \mathrm{mg} / \mathrm{m}^{2}$ daily $\times 3$ to daunorubicin $45 \mathrm{mg} / \mathrm{m}^{2}$ daily $\times 3$ (in combination with cytarabine) in younger (age $<60$ years) and older patients (age $60+$ years) ${ }^{6,7}$. In younger patients, 
high-dose daunorubicin was associated with a significantly higher $\mathrm{CR}$ rate $(71 \%$ versus $57 \% ; p<0.001)$ and longer survival (median 24 versus 16 months; $p=0.003$ ). However, the benefit was observed only in patients younger than 50 years and those with normal karyotypes ${ }^{7}$. In older patients, high-dose daunorubicin was associated with a higher CR rate $(64 \%$ versus $54 \% ; p=0.002)$ but not with improved survival, although a survival benefit was observed in the subset of patients 60 to 65 years old ${ }^{6}$. Daunorubicin $45 \mathrm{mg} /$ $\mathrm{m}^{2}$ daily $\times 3$ is sub-standard. Daunorubicin $60 \mathrm{mg} / \mathrm{m}^{2}$ daily $\times 3$ may be as effective and less toxic than $90 \mathrm{mg} / \mathrm{m}^{2}$ daily $\times 3$. A French study analyzed 402 patients (median age 49 years) who received daunorubicin $60 \mathrm{mg} / \mathrm{m}^{2}$ versus $90 \mathrm{mg} / \mathrm{m}^{2}$ as part of $3+7$ induction, and reported no difference in CR, induction mortality, RFS or overall survi$\mathrm{val}^{103}$. A MRC study compared daunorubicin $60 / \mathrm{m}^{2}$ versus $90 \mathrm{mg} / \mathrm{m}^{2}$ during induction and reported no difference in the longer term outcome (2-year survival $60 \%$ versus $59 \%$; $p$ $0.14)$, but a higher early mortality with daunorubicin $90 \mathrm{mg} /$ $\mathrm{m}^{2}{ }^{204}$. These studies helped establish daunorubicin $60 \mathrm{mg} /$ $\mathrm{m}^{2}$ daily $\times 3$ as the likely optimal dose schedule of daunorubicin.

Studies comparing idarubicin to daunorubicin, including a meta-analysis of five randomized trials, indicated that idarubicin may be associated with higher CR and survival rates ${ }^{105}$. Pautas and colleagues ${ }^{106}$ randomized 468 patients to induction therapy of standard-dose cytarabine in combination with daunorubicin $80 \mathrm{mg} / \mathrm{m}^{2}$ daily $\times 3$ versus idarubicin $12 \mathrm{mg} / \mathrm{m}^{2}$ daily $\times 3$ or 4 days. Idarubicin for 3 days resulted in a higher CR rate $(83 \%$ versus $70 \% ; p=0.007)$ and a trend for better 4 -year EFS ( $21 \%$ versus $12 \%)$ and survival (32\% versus $23 \%$ ) rates. A four-day schedule of idarubicin was not better. A retrospective analysis of two large French trials comparing idarubicin to daunorubicin in 727 patients reported that idarubicin $12 \mathrm{mg} / \mathrm{m}^{2}$ daily for 3 days resulted in significantly higher CR $(69 \%$ versus $61 \% ; p=0.03)$ and cure rates $(16.6 \%$ versus $9.8 \% ; p=0.018)$ compared with daunorubicin ${ }^{107}$. Mandelli and the Italian colleagues ${ }^{108}$ randomized 2157 patients to daunorubicin $\left(50 \mathrm{mg} / \mathrm{m}^{2}\right.$ daily $\times 3)$, idarubicin $\left(10 \mathrm{mg} / \mathrm{m}^{2}\right.$ daily $\left.\times 3\right)$, or mitroxantrone $\left(12 \mathrm{mg} / \mathrm{m}^{2}\right.$ daily $\left.\times 3\right)$, in combination with standarddose cytarabine. Both idarubicin and mitroxantrone were associated with higher 5-year RFS (37\% versus 29\%; $p=$ 0.02 ) and survival rates ( $43 \%$ versus $45 \%$ versus $36 \%$; $p=$ 0.01 ) among patients who did not receive allogeneic SCT. At MD Anderson, we use idarubicin $8-10 \mathrm{mg} / \mathrm{m}^{2}$ daily $\times 3$ as part of the FAI/CLIA AML frontline regimens.

\section{Gemtuzumab ozogamicin}

Antibody-targeting therapy is a major success story in hematologic malignancies, particularly in lymphoid malignancies (antibodies targeting CD20, CD19, and CD22 in lymphomas, chronic lymphocytic leukemia, acute lymphoblastic leukemia). The development of GO, a CD33 monoclonal antibody bound to calicheamicin, has had a rough journey in AML. The Food and Drug Administration (FDA) originally granted accelerated approval of GO $\left(9 \mathrm{mg} / \mathrm{m}^{2}\right.$ on Days 1 and 15) in the US in May 2000 for the treatment of older patients (60 years or older) in first relapse who are not candidates for cytotoxic chemotherapy. This approval was based on three phase 2 studies in 142 patients with relapsed AML (response rate $30 \%$; CR rate $16 \%)^{109}$. The approval was conditional on a future demonstration of the GO benefit in randomized trials. Several studies then explored lower and fractionated dose GO schedules in frontline randomized trials $\left(3 \mathrm{mg} / \mathrm{m}^{2} \times 1\right.$ during induction and consolidation; $3 \mathrm{mg} / \mathrm{m}^{2}$ on Days 1,4 , and 7 during induction). The pivotal trial in the $\mathrm{US}^{16}$ randomized patients to standard $3+7$ with daunorubicin $60 \mathrm{mg} / \mathrm{m}^{2}$ daily $\times 3$, versus the addition of GO $6 \mathrm{mg} / \mathrm{m}^{2}$ on Day 4 to $3+7$, but with daunorubicin $45 \mathrm{mg} / \mathrm{m}^{2}$ daily $\times 3$ (equitoxic but suboptimal dose in retrospect). They reported a higher induction mortality rate with $\mathrm{GO}$ (5\% versus $1 \%$ ), which resulted in the withdrawal of GO from the US market in $2010^{16}$. This study had an unusually low mortality rate in the standard arm (usually about 3-7\%), which suggested that GO may have increased mortality. The dose of daunorubicin in the GO arm was suboptimal, as confirmed today by several studies (discussed earlier). Four other randomized trials later matured, all demonstrating the benefit of adding GO, either overall or in subsets of patients $^{15,98,110,111}$. A meta-analysis of the five randomized trials involving 3,325 patients showed that the addition of GO did not increase the CR rate, reduced the risk of relapse $(p=0.0001)$, and improved the 5-year survival rate $(p=0.01)$. The $\mathrm{GO}$ effect was most pronounced in AML with favorable cytogenetics (increased 5 -year survival rate from 50 to $75 \% ; p=0.0006$ ) and intermediate cytogenetics $(p=0.005)$. Gemtuzumab $3 \mathrm{mg} / \mathrm{m}^{2}$ was associated with fewer early deaths than $6 \mathrm{mg} / \mathrm{m}^{2}$ and provided equal efficacy ${ }^{17}$. This resulted in the FDA re-approval of GO at the lower dose schedules for AML therapy in $2017^{112,113}$.

\section{French experience with lomustine in older AML on $3+7$}

In three French studies involving 847 older patients ( $>60$ years), the investigators reported that the addition of lomustine (alkylating agent) $200 \mathrm{mg} / \mathrm{m}^{2}$ orally on Day 1 to idarubicin + cytarabine $(n=508)$, compared with idarubicin + cytarabine $(n=339)$, was associated with a higher CR rate (68\% versus $58 \% ; p=0.002)$, a similar rate of toxic deaths, and a longer survival (median 12.7 versus 8.7 months; $p=0.004$ ). By multivariate analysis, lomustine was an independent favorable treatment variable for achievement of $\mathrm{CR}(p=0.002)$ and for survival prolongation $(p=0.002)^{114}$. 


\section{The MD Anderson approach in 2020}

To summarize, the optimal frontline therapy for younger patients with AML is evolving. While many AML experts (and community oncologists) favor $3+7$ as the standard of care, better regimens may have emerged. These incorporate high-dose cytarabine during induction and consolidations, include nucleoside analogs into the regimens, may incorporate lower-dose $\mathrm{GO}$ as part of induction-consolidation in $\mathrm{CBF}$ and intermediatekaryotype AML, may add other targeted therapies, particularly FLT3 inhibitors (e.g., gilteritinib, midostaurin, sorafenib) in FLT3-mutated AML, and may add venetoclax to regimens in non FLT3- mutated AML (discussed later).

AT MD Anderson, younger patients with AML referred today are treated with a combination of idarubicin, highdose cytarabine and an adenosine nucleoside analog (fludarabine-FAI/FLAG-IDA; cladribine-CLIA). FLT3 inhibitors (gilteritinib, quizartinib) are added to the regimen in patients with FLT3-mutated AML. Venetoclax shorter courses (7-14 days) are under investigation in combination with FAI or CLIA in the other AML subsets ${ }^{15,116}$. Once in $\mathrm{CR}$, and based on availability of donors, patient age and comorbidities, pretreatment AML characteristics (cytogenetics, molecular profiles) and MRD status in CR, patients may be offered allogeneic SCT. On average, patients are considered for allogeneic SCT in first CR if they have high-risk disease based on adverse cytogenetic abnormalities, high FLT3mutation $\mathrm{AR}$, or persistent $\mathrm{MRD}>0.1 \%$ in $\mathrm{CR}$ post first consolidation. Otherwise, they complete 4-6 courses of consolidation and are then offered maintenance therapy with azacitidine and venetoclax for $2+$ years, with or without the addition of targeted inhibitors (e.g., FLT3 inhibitors if FLT3-mutated AML; IDH inhibitors if IDH-mutated AML). Figure 4 shows the approaches in community practice and at MD Anderson. Patients 50 years or older are offered induction therapy in the protected environment to reduce induction mortality (Table 4). In community practice, reasonable isolation procedures could be proposed: laminar airflow rooms; reverse isolation; gloves, masks, gowns; no plants or flowers; limiting visitors. Intensive supportive care is offered with antibiotic prophylaxis including antifungals (posaconazole or voriconazole) $)^{117,118}$.

With this general approach, the CR rate among nonselected younger patients with AML is $70-80 \%$, and the long-term survival rate is $40-50 \%$ (Fig. 1). With the encouraging data incorporating venetoclax, FLT3 inhibitors, IDH inhibitors, and monoclonal antibodies (GO, novel CD33 monoclonal antibodies), combined modality strategies involving targeted agents and chemotherapy are becoming a reality in the management of all younger and older patients with AML.

Since the 2015 AML review ${ }^{1}$, many of the strategies listed then as investigational are now FDA approved and used as standards of care, either in the FDA approved indications, or in combined modality therapies that synergize their clinical benefits and render them more cost-effective. This is certainly the case for venetoclax, FLT3 inhibitors (gilteritinib), and IDH inhibitors (enasidenib, ivosidenib). Next, we summarize such ongoing studies with intensive chemotherapy in younger patients with AML.

\section{Regimens with venetoclax}

At MD Anderson, the frontline regimens, FLAG/IDA and CLIA, are now combined with venetoclax for 7-14 days during induction and for 5-7 days in maintenance, as tolerated ${ }^{115}$. The preliminary data are encouraging. Among 28 patients treated with FLAG/ idarubicin-venetoclax, the overall response rate is $93 \%$, and the MRD negativity rate in CR $92 \%{ }^{115}$. Among 31 patients treated with CLIA-venetoclax, the overall response rate is $90 \%$, and the estimated 1-year survival $78 \%{ }^{116}$. The regimens are myelosuppressive as expected, but tolerable with very low rates of induction mortality. Growth factors and prophylactic antibiotics/antifungals are essential to reduce the risk and morbidity of opportunistic infections.

\section{Regimens with IDH inhibitors}

Stein and colleagues ${ }^{119}$ treated 134 patients with de novo AML and IDH mutations using a combination of $3+7$ and ivosidenib (IDH1 mutation; $n=60$ ) or enasidenib (IDH2 mutation; $n=91$ ). With $3+7+$ ivosidenib the overall response rate was $93 \%$ and the estimated 1year survival rate $79 \%$. With $3+7+$ enasidenib the overall response rate was $73 \%$ and the estimated 1-year survival rate $75 \%{ }^{119}$. The HOVON and German study groups are currently evaluating $7+3$ with either ivosidenib or enasidenib (versus placebo) in a large Phase III randomized study (NCT03839771).

\section{Regimens with FLT3 inhibitors}

Stone and colleagues ${ }^{120}$ conducted a randomized phase III RATIFY trial (CALGB 10603) in 717 patients $<60$ years of age with newly diagnosed FLT3-mutated AML (FLT3ITD and/or FLT3-TKD; median age 48 years; range 18 to 60 years) with the combination of $3+7$ with or without midostaurin. Seventy-seven percent of patients had a FLT3-ITD mutation and 23\% had a FLT3-TKD mutation. The addition of midostaurin improved the CR rate $(59 \%$ versus $54 \%, p=0.045$ ) and the survival (median survival 74.7 versus 25.6 months, $p=0.009$; estimated 5 -year survival rate $50 \%$ versus $42 \%$ ). The benefit was noted in FLT3-ITD low AR (AR less or equal 0.70), FLT3-ITD high AR (AR >0.70) and TKD-mutated AML. At MD Anderson, a matched-cohort analysis similarly showed the benefit of adding sorafenib to idarubicin-cytarabine in 


\section{A) The 3+7 Standard of Care}

Therapy of AML-The 3+7 Standard Approach

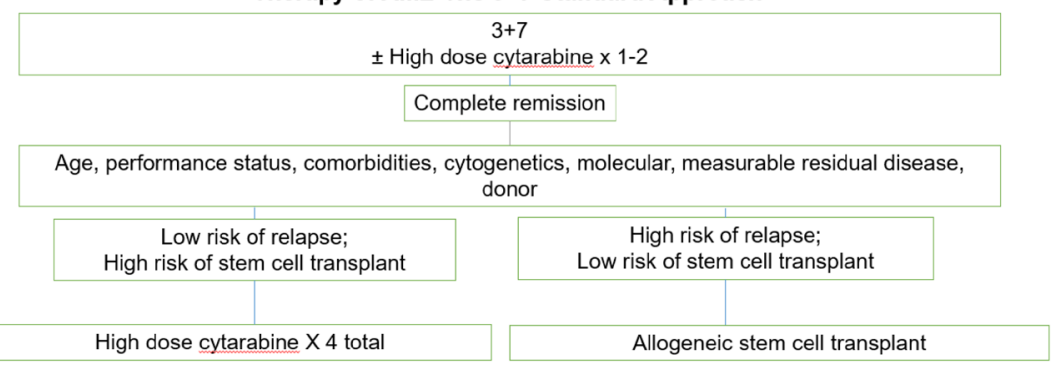

No maintenance

B) The MD Anderson Approach in Younger /Fit Patients

Therapy of Younger AML at MD Anderson in 2020+

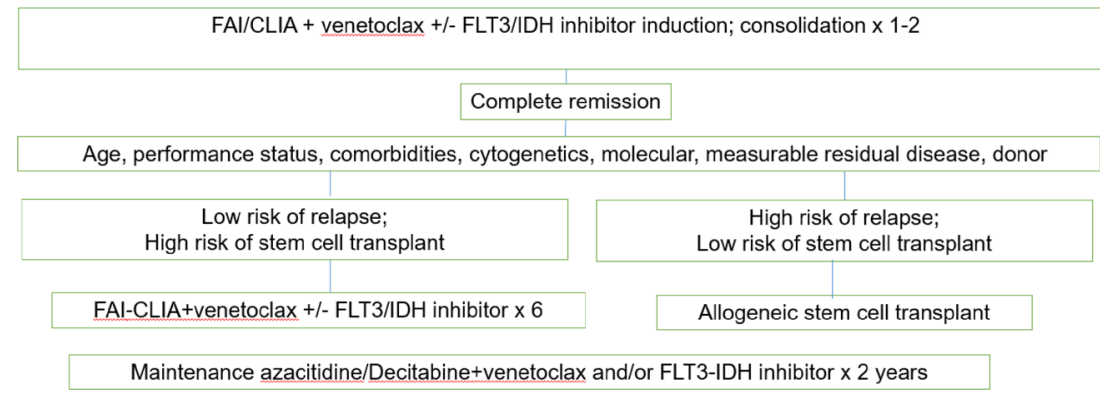

C) The MD Anderson Approach in Older/Unfit Patients

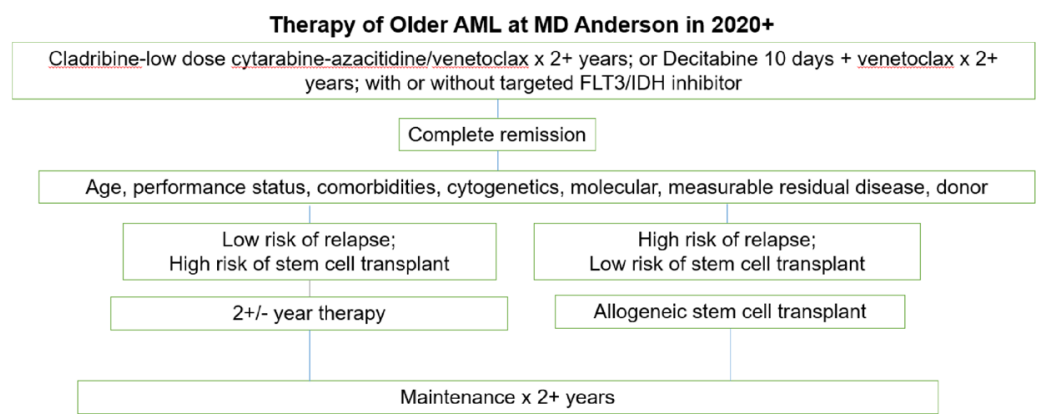

Fig. 4 Therapy of AML. A standard of care; B MD Anderson approach in young/fit patients, and $\mathbf{C}$ in older patients.

FLT3-mutated $\mathrm{AML}^{41}$. In our study of CLIA + FLT3 inhibitor (sorafenib/midostaurin), the CR rate was $86 \%$ and the estimated 1-year survival $70 \%{ }^{121}$.

Several studies are now underway evaluating newer generation FLT3 inhibitors (gilteritinib, quizartinib, crenolanib) in combination with intensive chemotherapy. Pratz and colleagues ${ }^{122}$ treated 33 patients with newly diagnosed AML with $3+7$ plus gilteritinib, reporting a marrow CR rate of $80+\%$ and an estimated 2-year survival rate of $70 \%$. These encouraging data have led to two randomized studies of $3+7+$ gilteritinib versus $3+7+$ midostaurin in Europe (HOVON 156ML; NCT04027309) and the US (NCT03836209). A phase III, randomized study of $3+7+$ quizartinib versus $3+7$ in frontline FLT3-ITD AML completed enrollment; the results are expected in 2021 (QUANTUM-First, NCT02668653)

Sorafenib has been used as maintenance therapy post allogeneic SCT in FLT3-mutated AML in single arm and randomized trials, all showing survival and/or RFS benefits for the addition of sorafenib maintenance ${ }^{123,124}$. A 
Table 4 General approach to patients with AML at MD Anderson in 2020.

\begin{tabular}{|c|c|c|}
\hline Disease & Therapy and comments & $\begin{array}{l}\% \text { 5-year } \\
\text { survival }\end{array}$ \\
\hline APL & $\begin{array}{l}\text {-ATRA plus arsenic trioxide- } \\
\text {-GO added for high-risk APL or persistent molecular disease } \geq 2-3 \text { months } \\
\text { into CR }\end{array}$ & $80-90$ \\
\hline CBF AML & $\begin{array}{l}\text {-FLAG-GO induction }+4+6 \text { consolidations } \\
\text {-age } \geq 60 \text { years: adjusted dose FLAG-GO } \\
\text {-Intolerance to FLAG-GO: decitabine or azacitidine } \times 12 \text { (according to } \\
\text { molecular MRD) } \pm \text { targeted therapies (i.e., GO as tolerated); } \\
\text {-Monitor response with real-time } \mathrm{PPCR} \text { testing (goal: }>3 \text {-log reduction) }\end{array}$ & 80 \\
\hline AML in younger patients & $\begin{array}{l}\text {-FLAG-IDA, CLIA induction + } 6 \text { consolidations } \\
\text {-FLT3 ITD: add FLT3-inhibitor (gilteritinib on study) } \\
\text {-Clinical trials: venetoclax added to CLIA or FIA; } \\
\text {-Future: activity of FLT3 inhibitors regardless of FLT3 status; IDH inhibitors + } \\
\text { chemotherapy in patients with IDHI/2 mutations }\end{array}$ & $40-50$ \\
\hline $\begin{array}{l}\text { AML in older patients/unfit for intensive } \\
\text { chemotherapy (age > 60-70 years; 8-week mortality } \geq \\
\text { 20-30\%) }\end{array}$ & $\begin{array}{l}\text {-Cladribine plus low-dose cytarabine alternating with HMA } \\
\text {-Clinical trial: cladribine-low-dose cytarabine- azacitidine + venetoclax } \\
\text {-Clinical trial: decitabine } 10 \text { days + venetoclax } \\
\text {-"Triplet" combinations on clinical trials (mutation specific): } \\
\text { Decitabine/azacitidine + venetoclax + quizartinib/gilteritinib (FLT3-mutated) } \\
\text { Azacitidine + venetoclax + IDH inhibitor (IDH mutated) } \\
\text { Azacitidine + venetoclax + APR246 (TP53 mutated) } \\
\text { Azacitidine + venetoclax + magrolimab } \\
\text {-Other investigational agents }\end{array}$ & $20-30$ \\
\hline Allogeneic SCT & $\begin{array}{l}\text {-In CR1 if poor cytogenetics, or } \\
\text { FLT3 ITD high allelic ratio, or adverse mutations, or } \\
\text { MRD positive in CR, and low treatment related mortality of SCT procedure } \\
\text {-CR2 and beyond: all potential patients }\end{array}$ & \\
\hline Salvage therapy & $\begin{array}{l}\text {-CRD1 } \geq 12 \text { months: high-dose cytarabine-based regimens } \\
\text {-FLAG-IDA + Venetoclax on clinical trial } \\
\text {-CRD } 1<12 \text { months: phase } 1-2 \text { trials } \\
\text {-Always recheck for mutations (next-generation sequencing), particularly for } \\
\text { FLT3 and IDHI/2 mutations; if mutations then target-based therapy }\end{array}$ & \\
\hline Supportive measures & $\begin{array}{l}\text {-Antibiotic/antifungal prophylaxis } \\
\text {-Protected environment/reverse isolation if } \\
\text { age } \geq 50 \text { years }+ \text { intensive chemotherapy, or } \\
\text { if age } \geq 60 \text { years + low-intensity therapy }\end{array}$ & \\
\hline
\end{tabular}

APL acute promyelocytic leukemia, ATRA all-transretinoic acid, GO gemtuzumab ozogamicin, FLAG-Ida, FAI fludarabine, high-dose cytarabine, idarubicin, CLIA cladribine, high-dose cytarabine, idarubicin, HMA hypomethylating agent.

randomized study of gilteritinib versus placebo administered after allogeneic SCT in FLT3-mutated AML may help address more definitively the benefit and optimal use of FLT3 inhibitors in this setting. (BMT CTN 1506; ClinicalTrials.gov identifier: NCT02997202).

While FLT3 inhibitors are now established therapies in combination regimens in FLT3-mutated AML, it is of interest that several non-targeted chemotherapy strategies have also shown selective benefits in FLT3-mutated AML, including induction regimens containing high-dose cytarabine, cladribine and high-dose daunorubicin ${ }^{92,125,126}$.

\section{Older patients with acute myeloid leukemia (or younger patients not fit for intensive chemotherapy) \\ Intensive chemotherapy}

The median age of patients with AML is 68 years, but most of the experience with $3+7$ and intensive 
chemotherapy regimens is in younger patients, usually 60 years or younger. Older patients with AML tolerate intensive chemotherapy poorly. In the study by Lowenberg and colleagues ${ }^{6}$ evaluating $3+7$ with daunorubicin $45 \mathrm{mg} / \mathrm{m}^{2}$ versus $90 \mathrm{mg} / \mathrm{m}^{2}$ daily $\times 3$, among 813 selected patients 60 years and older (median age 67 years), the median survival was 7 to 8 months and the estimated 3-year survival rate was 20\%. The study reported an acceptable low early mortality rate of $11-12 \%$. Whether this mortality rate is replicable in unselected patients in oncology community practice is questionable.

The treatment of older patients with AML remains challenging. Acute myeloid leukemia in older patients carries a distinctly different disease biology associated with high risk and often complex karyotype, a high incidence of cytogenetic abnormalities involving monosomies 5 and 7 and chromosome 17 abnormalities, a high incidence of multiple mutations including TP53 (20+\%), and a high incidence of secondary/therapy-related AML (20 to $30 \%)$. Older patients have multiple co-morbidities (hypertension; diabetes; organ dysfunctions including cardiac, pulmonary and renal abnormalities) that result in poor tolerance to intensive chemotherapy and high early (4- to 8-week) mortality rates. In community practice (SEER data; 2010-2017) treating unselected older patients, the 4-week mortality is $24 \%$ among patients 60-69 years old and the 5-year survival 18\%. Among patients 70 years and older (45\% of all AML), the 4-week mortality is $44 \%$ and the 5 -year survival $4 \%$. Clearly neither intensive chemotherapy nor supportive/hospice care are acceptable options in older AML.

At MD Anderson, historical studies using intensive chemotherapy in older patients with AML (age 60-65 years or older) showed CR rates of 40-50\%, 4-8-week mortality rates of 26-36\%, median survivals of 4-6 months, and one -year survival rates of $<30 \%{ }^{127,128}$. By multivariate analysis, independent adverse factors predictive of early mortality with intensive chemotherapy were: age 75 years and older; adverse karyotype with three or more chromosomal abnormalities; presence of an antecedent hematologic disorder; poor performance status (ECOG 2-4); creatinine level $1.3 \mathrm{mg} / \mathrm{dl}$ or higher; and treatment outside a protected environment. The expected 8-week mortality was $10-19 \%$ with the presence of $0-1$ adverse factors, and $36-65 \%$ with the presence of 2-5 adverse factors ${ }^{127}$.

\section{Epigenetic and low-intensity therapy}

Faced with the poor results with intensive chemotherapy, investigators began in the 1990's evaluating lower-intensity strategies in patients unfit for intensive chemotherapy (expected high-early mortality). These included low-dose cytarabine, HMA therapy, and targeted therapies (monoclonal antibodies; more recently FLT3 inhibitors and IDH $1 / 2$ inhibitors). This raised the question of how to select patients unfit for intensive chemotherapy. At our institution, we use the above model to select such patients, based on an estimated early mortality rate in excess of $10 \%$. Over time and over several investigational studies since 2000, we have shown that lower-dose chemotherapy/HMA therapy combinations now provide, since 2015, overall response rates as high as with intensive chemotherapy, significantly lower rates of early mortality and myelosuppressionassociated complications, and survival equivalent or superior to intensive chemotherapy ${ }^{129,130}$.

In clinical practice, leukemia experts often base the decision of intensive versus low-intensity therapy on the "oculometer" (looking at the patient and deciding by intuition and experience). The approach is subjective and based on the oncologist's experience and perception of the patient's condition (performance, co-morbidities, infections at presentation, tolerance to intensive chemotherapy). This may be better replaced by more objective prediction models such as the one used at our institution. Patients are then categorized according to their predicted early mortality (based on the multivariate prognostic models) ${ }^{127,128}$. If the expected 4-8-week mortality is $<10 \%$, they are offered intensive chemotherapy. If it is $>10-20 \%$, they are offered low-intensity approaches. Of interest, a third of patients who present as afebrile with normal chest radiographs may have significant abnormalities detected by computerized tomography (CT) scans (infections, nodular lesions suggestive of early fungal pneumonia, bleeding, other) ${ }^{131}$. Patients with AML and pneumonia at diagnosis have a significantly higher risk of early mortality with intensive chemotherapy (4-8-week mortality 15-30\%; Sasakiunpublished). Future studies should investigate incorporating pretreatment routine $\mathrm{CT}$ of chest findings into predictive models of early mortality in AML.

Historically, many older patients (age 70 or older) with AML were offered supportive palliative or hospice care ${ }^{132}$. The MRC AML14 study randomized 217 older patients to low-dose cytarabine $20 \mathrm{mg}$ subcutaneously twice daily $x$ 10 days versus supportive care and hydroxurea ${ }^{133}$. Lowdose cytarabine was associated with a higher CR rate (18\% versus $1 \% ; p=0.00006$ ) and with longer survival (odds ratio: $0.60 ; p=0.0009$ ). This study drove home an important message: that an active tolerable treatment would have a significant effect on improving early mortality and overall survival, even among patients deemed suitable only for supportive care at the time of diagnosis. In the 2000s, studies with HMAs demonstrated the benefits of decitabine and azacitidine for the treatment of older patients unfit for intensive chemotherapy. A phase 3 study randomized 485 patients 65 years or older to decitabine $20 \mathrm{mg} / \mathrm{m}^{2}$ IV daily $\times 5$ every month versus supportive care or low-dose cytarabine. In a final analysis, the median survival was 7.7 with decitabine versus 
5 months with supportive care or low-dose cytarabine ( $p$ $=0.036$ ). This led to the European Medicines Agency (EMA) approval of decitabine for the treatment of older patients with $\mathrm{AML}^{26}$. A similar study (AZA-AML-001) randomized 488 older patients to azacitidine $(n=241)$ versus three predetermined conventional care regimens ( $n=247$; low-dose cytarabine, intensive chemotherapy, supportive care). Azacitidine therapy was associated with longer survival (median 10.4 versus 6.5 months; $p=0.06$; hazard ratio 0.85$)^{28}$.

Studies have also evaluated longer durations of decitabine schedules $\left(20 \mathrm{mg} / \mathrm{m}^{2} \text { daily } \times 10\right)^{134}$ in combinations (venetoclax, FLT3 and IDH inhibitors, others). The FDA approved recently a $100 \%$ absorbable oral formulation of decitabine plus oral cedazuridine (cytosine deaminase inhibitor; oral combination bioequivalent to intravenous decitabine $)^{135,136}$. This opens research into potentially highly effective oral therapies in older AML (oral decitabine-cedazuridine plus venetoclax), which may improve tolerance and quality of life, and offer safe and effective outpatient therapy.

At MD Anderson, prior to the discovery of the role of venetoclax in AML, we had evaluated sequential three-drug low-intensity therapy combining an adenosine nucleoside analog (clofarabine or cladribine) with low-dose cytarabine, and alternating this with decitabine over a period of 18 months ${ }^{137,138}$. Among 248 patients (median age 69; range $48-85$ years) treated with the two regimens, the overall response rate was $66 \%$, the CR rate $59 \%$, the early (4-week) mortality rate $2 \%$, the median survival 12.5 months, and the estimated 2-year survival rate $29 \%$. Among patients with normal karyotype, the median survival was 19.9 months and the estimated 2-year survival rate $45 \%{ }^{137,138}$. At that time, compared to single-agent HMAs, which were standard therapy, the triple-nucleoside analog (cladribine-cytarabineHMA) low-intensity therapy showed better results. It also represented a novel, well-tolerated, effective new backbone therapy upon which to build combination approaches. Deriving from the success of HMA + venetoclax combinations, we are evaluating the combination of cladribine-lowdose cytarabine-azacitidine with venetoclax in older AML.

\section{Regimens with hypomethylating agents and venetoclax (ABT-199)}

One therapeutic strategy to target AML involves activation of the intrinsic or mitochondrial pathway of apoptosis. This pathway is regulated by the BCL2-family of proteins. It involves a dynamic balance of pro-apoptotic effectors (Bak, Bax) and anti-apoptotic proteins (BCL-2, $\mathrm{BCL}-\mathrm{XL}, \mathrm{MCL}-1)$. In a balanced state, the anti-apoptotic proteins bind to and sequester the pro-apoptotic proteins and prevent them from triggering apoptosis. Antiapoptotic proteins are overexpressed in many tumors including AML. Small molecule "BH3 -mimetics" were developed that bind to the anti-apoptotic proteins in the BH3 domain and liberate pro-apoptotic proteins that subsequently trigger apoptosis. The earlier generation of BH3 mimetics bound efficiently to multiple anti-apoptotic proteins, including BCL-2, BCL-XL, and MCL-1, and thus were associated with unacceptable on- target toxicities, including thrombocytopenia.

Venetoclax (ABT-199; BCL2 inhibitor) was developed over many years as a more advanced $\mathrm{BH} 3$ mimetic molecule designed to retain specificity for BCL-2, but without affinity for BCL-XL or MCL-1. Venetoclax has already revolutionized the treatment of chronic lymphocytic leukemia and may have a role in other cancers (acute lymphoblastic leukemia, myelodysplastic syndrome, lymphoma and myeloma subsets). The AML blasts and AML stem cells depend on BCL-2 for survival, but normal hematopoietic stem cells depend on MCL-1. This presented the rationale for investigating venetoclax in AML. Preclinical studies confirmed its activity in AML cell lines, in murine primary xenografts, and in AML samples ${ }^{139}$. A phase 2 single-agent study in AML investigated venetoclax ( $800 \mathrm{mg}$ daily) in 32 patients with refractory-relapsed AML. The overall response rate was $15 \%$, with another $19 \%$ of patients having reductions of blasts ${ }^{140}$. Responses appeared to be more frequent among patients with $I D H$ mutations, a clinical observation that confirms preclinical studies, suggesting BCL-2 to be a synthetic lethal partner AML with IDH1/2 mutations ${ }^{53,140}$.

Based on the encouraging preclinical data of venetoclax in combination with HMAs and low-dose cytarabine, single-arm trials evaluated these combinations in newly diagnosed patients with AML who were older than 75 years or unfit to receive intensive chemotherapy. The positive results (overall response rates 67\%; estimated median survival 17.5 months; 2-year survival rate $40 \%$ ) led to the FDA accelerated approval of venetoclax in combination with epigenetic therapy or low-dose cytarabine for the treatment of these patients ${ }^{141,142}$.

The subsequent VIALE-A pivotal trial randomized such patients $(75+$ years; or unfit for intensive chemotherapy) to therapy with azacitidine alone or in combination with venetoclax. Among 431 patients randomized on a 2:1 basis to azacitidine plus venetoclax ( $n$ $=286)$ or azacitidine $(n=145)$, the addition of venetoclax resulted in a significantly longer survival (median survival 14.7 versus 9.6 months; $p<0.001$ ). The overall response rate $(66.4 \%$ versus $28.3 \% ; p<0.001)$ and $C R$ rate (29.7\% versus $17.9 \% ; p<0.001)$ were also higher ${ }^{143}$. A similar randomized study (211 patients; 2:1 randomization) of low-dose cytarabine with venetoclax versus lowdose cytarabine alone showed a median survival of 8.4 versus 4.1 months $(p=0.04)$, an overall response rate of $48 \%$ versus $13 \%(p<0.001)$, and a CR rate of $27 \%$ versus $7 \%(p<0.001)^{144}$. 
A single-arm trial from our institution investigated the use of decitabine for a 10-day induction with venetoclax (followed by maintenance with monthly decitabine for 5 days and venetoclax for 14-21 days). Among 70 older patients (median age 72 years; range 70-78 years) with newly diagnosed de novo AML treated with the regimen, the overall response rate $(\mathrm{CR}+\mathrm{CRi})$ was $84 \%$, the $\mathrm{CR}$ rate $67 \%$, the 4 -week mortality rate $0 \%$, and the median survival 18.1 months $^{145}$.

\section{Experience with low-intensity chemotherapy combination and venetoclax}

One of the current frontline trials in older AML at MD Anderson explores the combination of cladribinecytarabine-venetoclax alternating with azacitidinevenetoclax $^{146}$. Among 48 patients treated so far (median age 68 years; range $57-84$ years), the CR rate was $77 \%$, the overall response rate $94 \%$, the MRD negativity rate $80 \%$, the 4 -week mortality rate $0 \%$, and the estimated 1 -year survival rate $70 \%$.

\section{Hypomethylating agents with FLT3 inhibitors}

The combination of azacitidine and sorafenib in older patients with FLT3-ITD AML resulted in a CR-CRi rate of $78 \%$ and a median survival of 8.3 months ${ }^{147}$.

Gilteritinib was combined with azacitidine in the frontline setting in the ongoing phase III LACEWING trial. The initial safety run-in data from this study showed an encouraging marrow CR rate of $67 \%$ among the first 15 patients treated prior to the beginning of the randomization $^{148}$. A gilteritinib dose of $120 \mathrm{mg}$ daily was selected with standard-dose azacitidine (NCT02752035).

Older patients (age 65 years or older) and patients not fit for intensive chemotherapy (based on predicted high early mortality) are now offered low-intensity strategies using combinations of cladribine and low-dose cytarabine alternating with decitabine or azacitidine together with venetoclax; decitabine (10-day induction, 5-day maintenance) combined with venetoclax, and other HMAs (e.g., oral decitabine) plus venetoclax-based combinations that also incorporate FLT3 inhibitors (if FLT3-mutated AML), IDH inhibitors (if IDH-mutated AML), or APR246 or magrolimab (if TP53-mutated AML). (Table 4).

\section{CPX-351 in older AML}

CPX-351 is a nano-scale liposome, which contains a fixed 5:1 molar ratio of cytarabine and daunorubicin ${ }^{149}$. Following the encouraging preclinical and phase 1-2 trials in the subset of secondary AML,a pivotal phase 3 randomized trial in newly diagnosed secondary AML accrued 309 patients randomized to CPX-351 versus $3+7$. Therapy with CPX-351 was associated with a significantly longer survival (hazard ratio $0.69 ; p=0.005$ ). The $\mathrm{CR}$ rate was $38 \%$ with CPX-351 versus $26 \%$ with $3+7(p=0.035)$; the CR + CRi rate was $48 \%$ versus $33 \%(p=0.016)$. CPX351 was associated with a longer duration of myelosuppression. More of the patients achieving CR post CPX-351 were able to undergo later allogeneic SCT (20\% versus $12 \%)$; their survival was also longer post SCT. The study findings resulted in the FDA approval of CPX-351 as frontline therapy of secondary AML ${ }^{150,151}$. Ongoing studies are combining CPX-351 with venetoclax, GO, and other targeted therapies.

\section{Glasdegib}

The Hedgehog $(\mathrm{Hh})$ signaling pathway plays critical roles in embryogenesis and stem cell maintenance. Dysregulations in the $\mathrm{Hh}$ pathway can result in the development, maintenance and expansion of the leukemic stem cells, which play a critical role in AML pathogenesis, persistence and progression $^{152}$.

Glasdegib, a cyclopamine derivative, is a selective inhibitor of Smoothened (SMO), a component of the $\mathrm{Hh}$ signaling pathway. Following encouraging preclinical and phase 1-2 trials, a phase 2 study investigated low-dose cytarabine alone versus low-dose cytarabine plus glasdegib $100 \mathrm{mg}$ daily. The addition of glasdegib was associated with a significant prolongation of survival (median survival 8.8 months versus 4.9 months; 12-month survival $59.8 \%$ versus $38.2 \%)^{153}$. This led to the approval of glasdegib for the treatment of newly diagnosed AML in patients $75+$ years old or unsuitable for intensive induction chemotherapy ${ }^{154}$. Ongoing studies are evaluating glasdegib combination with azacitidine and with intensive chemotherapy.

\section{The potential roles of APR-246 and magrolimab in TP53- mutated AML}

TP53- mutated AML is associated with older age, therapy-related disease, complex (adverse) cytogenetics, and very poor prognosis. Even with the advent of HMAs plus venetoclax, older patients with TP53-mutated AML ineligible for induction therapy continue to do poorly: response rates $50 \%$ but median survival only 3-6 months ${ }^{145}$.

APR-246 is a novel agent that may restore the transcriptional activity of unfolded wild-type or mutant $p 53$, leading to induction of apoptosis in cancer cells with mutant $p 53^{155}$. In two parallel ongoing studies in France and the US, the combination of azacitidine with APR-246 produced $\mathrm{CR} / \mathrm{CRi}$ rates of $60-80 \%$; $>60 \%$ of responders had undetectable TP53 mutation by next-generation sequencing ${ }^{156,157}$. A phase III randomized study of azacitidine with or without APR-246 in frontline MDS and AML with 20 to $30 \%$ blasts has recently completed enrollment (NCT03745716), and reported not to have met the study primary endpoint of significantly higher CR rate (December 28, 2020). 
CD47 functions as a macrophage checkpoint, providing a potent "do not eat me" signal that allows tumor cell evasion and immune destruction by macrophages. CD47 is upregulated in AML, and CD47 upregulation was independently associated with a poor prognosis ${ }^{158-160}$. Hu5F9-G4 (magrolimab) is a humanized monoclonal antibody that binds CD47 and blocks it from interacting with its ligand SIRPa on phagocytic cells, leading to phagocytic elimination of cancer cells. The combination of magrolimab plus azacitidine was evaluated in patients with newly diagnosed AML who were unfit for intensive chemotherapy or who had MDS intermediate-higher risk (Revised International Prognostic Scoring System [IPSSR]). In 34 evaluable patients with AML, the objective response rate was $65 \%$, (CR $40 \%$, CRi $12 \%$ ). The median time to response was 2.0 months. Among patients who had abnormal cytogenetics at baseline, $47 \%$ achieved complete cytogenetic response. In patients harboring TP53 mutations, the overall response rate was 71\% (15 of 21 patients) and the CR rate $48 \%$ (5 of 12 patients). The median survival in TP53-mutant AML was 12.9 months and in TP53 wild-type 18.9 months $^{161}$.

\section{Maintenance therapy in acute myeloid leukemia}

Maintenance therapy is an established positive approach in many cancers, including acute lymphocytic leukemia. However, studies in AML could not confirm a clear benefit of maintenance therapy, until the recent positive results reported with oral azacitidine (CC-486). The oral drug is poorly absorbed (AUC $10-30 \%$ of intravenous azacitidine). In an international multi-center trial (QUAZAR AML-001), 472 patients 55 years and older (median age 68 years) with AML in first CR for $<4$ months were randomized to oral azacitidine (CC-486) $300 \mathrm{mg}$ orally daily $\times 14$ every month $(n=238)$, or placebo $(n=234)$. The median survival was 24.7 months with CC486 versus 14.8 months with placebo (hazard ratio 0.69, $p=0.0009$ ). The median RFSs were 10.2 and 4.8 months. The FDA approved CC-486 as oral maintenance therapy for this indication in September $2020^{162}$.

A second study (HOVON97) randomized 116 patients 60 years and older with AML who were in CR post two courses of intensive chemotherapy to azacitidine $50 \mathrm{mg} / \mathrm{m}^{2}$ subcutaneously daily $\times 5$ every month for 12 courses $(n=56)$ versus observation $(n=60)$. The 12 -month disease-free survival (DFS) was $64 \%$ with azacitidine versus $42 \%$ with observation $(p=0.04)^{163}$

In the context of post-SCT, maintenance therapy has also been of benefit. Buchert and colleagues ${ }^{124}$ reported on 83 patients (median age 54 years) with FLT3-ITD AML post allogeneic SCT who were randomized to sorafenib 200-400 mg twice daily for two years versus placebo. The 2 -year PFS rate was $85 \%$ with sorafenib versus $53 \%$ with placebo $(p=0.04)$. Survival was also better (hazard ratio
0.447; $p=0.03$ ). In the pivotal gilteritinib versus salvage chemotherapy (ADMIRAL) trial in 371 patients with FLT3-mutated AML, Perl and colleagues ${ }^{164}$ reported on 51 patients achieving a response post gilteritinib and undergoing allogeneic SCT who either resumed gilteritinib post SCT $(n=35)$ or did not $(n=16)$. The median survival was longer with gilteritinib resumption (16.2 months versus 8.4 months; hazard ratio $0.387 ; p=$ $0.024)$.

\section{Translating the published literature into real- world experience}

Here a word of caution - an analysis of the SEER data (more reflective of the reality on the ground and of general oncology community practice) in about 29,000 patients with AML showed results substantially worse than those reported from single institutions and from cooperative trials. In the SEER data, the results have improved since 2000 in APL (5-year survival about $60+\%$ ) and CBF AML (5-year survival 50\%), mostly in patients younger than 60 years. However, even restricting the analysis to the Years 2000-2017, the 4-week mortality among patients 40-59 years old with de novo AML (excluding APL and CBF AML) is $27 \%$ and the 5 -year survival rate $40 \%$. Among patients $70+$ years old, the 4-week mortality rate is $45-50 \%$ and the 5 -year survival rate $<5 \%{ }^{85}$.

Therapy of AML is difficult and requires long-term expertise. This is because AML is rare, and often affects older patients who require chemotherapy in the setting of a compromised marrow by the disease; this results in severe cytopenias at diagnosis and throughout therapy. All these conditions require also the use of antibiotics prophylaxis and the prompt availability of optimal supportive care (platelets and blood transfusions; skilled emergency centers and facilities to deliver the support needed, recognize infections and sepsis, implement proper broadspectrum IV antibiotics, and offer timely intensive care unit care when needed). Thus, the risks of serious morbidities, mortality and treatment abandonment are high.

For a long time, it was assumed that AML care may be equally optimal in the community practice as it is in published data from cooperative trials. This, however, may not be the case. In several AML cooperative trials, the early (4-week) mortality with intensive chemotherapy in younger patients with AML ranges from 1 to $10 \%{ }^{91,165}$. At our institution, the early mortality with intensive chemotherapy is $<5 \%$; the early mortality with lowintensity regimens in older AML is $1-2 \%$.

Two recent studies reported significantly higher early mortality rates among patients treated in non-academic versus academic centers, and in non- $\mathrm{NCI}$-designated versus NCI-designated cancer centers ${ }^{166,167}$. In a National Cancer data Base of 60,738 patients with AML, the 1 -month mortality was $16 \%$ in academic centers and 
$29 \%$ in non-academic centers $(p<0.001)$, and the 5 -year survival rate was $25 \%$ versus $15 \%(p<0.001)^{166,167}$. The second study from California in 7007 patients with AML reported an early mortality rate in AML of $12 \%$ in NCIdesignated cancer centers versus $24 \%$ in non-NCIdesignated cancer center ${ }^{167}$.

Perhaps AML, being rare and requiring intensive chemotherapy and supportive care in the setting of a compromised marrow, is better treated in specialized leukemia centers, rather than in the community practice.

\section{Allogeneic and autologous stem cell transplantation}

A meta-analysis combining data of multiple randomized trials demonstrated the significant benefit, on average, of allogeneic SCT in first $\mathrm{CR}^{168}$. The value of allogeneic SCT in AML first CR was difficult to confirm in earlier randomized trials because of: (1) the limited number of patients in each study (may not detect modest but clinically significant benefits); (2) the lead time bias to allogeneic SCT; (3) many patients allocated to allogeneic SCT could not undergo the SCT (infections, organ dysfunction, new chemotherapy related morbidities, AML relapse, others); (4) patients allocated to chemotherapy in first CR may have benefited from an allogeneic SCT in second CR. A study by the MRC reported that the benefits of chemotherapy versus allogeneic SCT in first CR were similar when the benefit of allogeneic SCT in second CR was factored in ${ }^{169}$.

Allogeneic SCT is an accepted standard of care in first $\mathrm{CR}$, and based on several patient, AML and treatmentassociated factors: (1) the presence of an adverse AML karyotype or high FLT3-mutated AR at diagnosis; (2) persistent MRD in CR; (3) low-risk of SCT-associated mortality based on the patient's age and co-morbidities, donor availability and degree of matching. With the FDA approval and availability of venetoclax and FLT3 and IDH inhibitors, the role of allogeneic SCT in first CR needs to be continuously evaluated.

Allogeneic SCT should not be considered as a one-time independent procedure, but part of the total strategy of chemotherapy-targeted therapy-SCT. Investigations of post allogeneic SCTmaintenance strategy to reduce the risk of relapse should be incorporated into this continuum, including azacitidine-decitabine (parenteral and oral), FLT3 inhibitors, IDH inhibitors, venetoclax, and others.

Autologous SCT has been largely abandoned in the United States because of the lack of a definite benefit. European AML experts still advocate for its role in first CR based on randomized trials showing that autologous SCT provides equivalent results to multiple chemotherapy consolidations (usually fewer than 4). With the knowledge concerning persistence of MRD in CR, it is possible that historical studies may have reinfused autologous marrows with significant persistent AML disease burden, thus perhaps increasing the relapse rates. This may have abrogated the potential benefit of this approach. Future studies may evaluate again the benefit of autologous SCT using collected MRD-negative marrows. At our institution, autologous SCT is still considered occasionally in the setting of APL and CBF AML in second CR and with negative molecular MRD in collected stem cells.

\section{Salvage therapy}

The choice of salvage therapy in AML depends on multiple factors: patient age and wishes, co-morbidities, salvage status, prior therapies, duration of prior response, exposure to allogeneic SCT, leukemia characteristics, and availability of investigational therapies. Guidelines of salvage therapies offered at our institution are detailed below.

In young/fit patients with AML and failure or progression on $3+7$ regimens, therapies that include high-dose cytarabine provide good results. Using the FLAG-IDA plus venetoclax regimen in 25 patients in Salvage 1, the marrow $\mathrm{CR}$ rate was $65 \%$ and 1 -year survival $52 \%{ }^{115}$. The combination of HMA therapy (azacitidine, decitabine) plus venetoclax may help patients not previously exposed to either agent. For patients post frontline high-dose cytarabine-based regimens (FAI-FLAG/IDA, CLIA) who are in first relapse with a first CR duration of 12 months or longer, we still offer the high-dose cytarabine-based regimens (FLAG-IDA, CIA, CLIA, twice daily fludarabine + cytarabine $)^{170}$ in combination with novel targeted therapies as indicated (venetoclax, FLT3 or IDH inhibitors). In salvage situations, repeating the molecular studies for FLT3, IDH 1-2, and TP53 mutations may identify the emergence of resistant clones with these mutations. Patients may then become candidates for targeted inhibitors-based therapies. Patients in second salvage or beyond are offered phase 1-2 investigational approaches.

Patients achieving subsequent CR should be considered for allogeneic SCT immediately, provided they understand the procedure risks, expected mortality rates, and expected (low) rate of long-term survival. Along these lines, we are investigating a regimen of sequential intensive chemotherapy, with the application of allogeneic SCT at the time of marrow aplasia (Day 21-35 of chemotherapy) rather than after achievement of CR (which is of low probability; $<10-20 \%$ in most such situations).

\section{FLT3 inhibitors in AML salvage}

Gilteritinib (SP 2215) is a potent type-1 FLT3 inhibitor (dual FLT3-AXL inhibitor) with excellent selectivity against FLT3 mutations (both FLT3-ITD and FLT3-TKD mutations). Gilteritinib $120 \mathrm{mg}$ daily produced CRc (composite CR) rates of $45-50 \%$ as a single agent in 
relapsed/refractory FLT3-mutated AML patients ${ }^{171}$. The phase 3 pivotal ADMIRAL trial randomized (2:1) 371 patients with relapsed FLT3-mutated AML to gilteritinib $120 \mathrm{mg}$ daily $(n=247)$ or investigator choice salvage chemotherapy (both high- and low-dose chemotherapy) $(n=124)^{164}$. Gilteritinib therapy resulted in a significantly longer survival (median survival 9.3 versus 5.6 months; hazard ratio $0.637 ; p=0.0007$ ). It was also associated with a higher rates of CR ( $21 \%$ versus $11 \%$; $p=0.013), \mathrm{CR} / \mathrm{CRh}$ rate $(34 \%$ versus $15 \%)$, and CRc rate $(54 \% \text { vesus } 22 \%)^{164}$. This led to the FDA approval of single-agent gilteritinib as salvage therapy of FLT3-mutated AML. Ongoing studies are combining gilteritinib with HMA therapy and with intensive chemotherapy, as well as with venetoclax in frontline, salvage, and maintenance strategies in AML.

Combination therapy with agents that induce apoptosis may enhance cytotoxicity against FLT3-mutated and wild-type clones and potentially delay or prevent drug resistance to FLT3 inhibitor-based therapies. Preclinical data indicated strong synergism between venetoclax and FLT3 inhibitors. An ongoing phase IB study is evaluating the combination of venetoclax and gilteritinib (NCT03625505) in refractory-relapsed AML (most patients with prior exposure to FLT3 inhibitors). Currently, 31 of 37 patients (84\%) treated achieved marrow $\mathrm{CR}$; the median duration of response has not been reached $^{172}$. A triplet-therapy combining azacitidine, venetoclax and gilteritinib in older AML is ongoing.

\section{Isocitrate dehydrogenase inhibitors in AML salvage}

The IDH 1-2 mutations induce neomorphic IDH enzyme activity, which results in aberrant production of the onco-metabolite 2-hydroxyglutarate (2-HG). The 2HG competitively inhibits alpha-ketoglutarate $(\alpha K G)$, and leads to dyregulated epigenetic function, a hypermethylated phenotype, and a block in maturation, leading to AML tumorigenesis ${ }^{173}$.

Enasidenib, formerly AG221, is an orally bioavailable small molecule inhibitor of mutant $I D H 2$, which is FDA approved for the treatment of relapsed-refractory $\mathrm{IDH} 2$ mutated AML at a dose of $100 \mathrm{mg}$ orally continuously daily. The FDA approval was based on the results of the Phase1-2 trial in 176 patients with relapsed-refractory IDH2-mutated AML. Enasidenib therapy resulted in an overall response rate of $41 \%$, a CR/CRh rate of $23 \%$, a median response duration of 5.8 months, and a median survival of 9.3 months. When used as monotherapy, patients with RAS pathway co-mutations and/or high mutational burden ( $>6$ mutations) were less likely to respond $^{173,174}$, suggesting the importance of combination therapy (under evaluation in both newly diagnosed and relapsed IDH2-mutated AML). In a randomized Phase 2 study in newly diagnosed IDH2-mutated AML of azacitidine + enasidenib compared with enasidenib alone, the combination resulted in a significantly higher $\mathrm{CR}$ rate (53\% versus $12 \%)$ and overall response rate $(71 \%$ versus $42 \%$ ), and a trend for improved EFS (17 months versus 11 months). The overall median survival was impressive, 22 months, but similar in both arms, likely because of the availability of effective salvage ${ }^{175}$.

Ivosidenib, formerly AG120, is a selective small molecule inhibitor of $I D H 1$. Ivosidenib $500 \mathrm{mg}$ daily was approved by the FDA for the treatment of relapsedrefractory IDH1-mutated AML (as well frontline therapy of IDH1-mutated AML in patients unfit for intensive chemotherapy) based on the results of the Phase 1-2 clinical trial evaluating 179 patients. In this study, ivosidenib produced an overall response rate of $42 \%$, a CR/ CRh of $30 \%$, a CR of $22 \%$, and a median survival of 8.8 months $^{176}$. Similar to enasidenib, mutations in the RTK pathway (i.e., RAS, PTPN11 and FLT3 mutations) were associated with a lower response rate to ivosidenib monotherapy ${ }^{177}$. A trial of ivosidenib + venetoclax + azacitidine is currently ongoing in newly diagnosed and relapsed IDH1-mutated AML.

\section{Expanding on topics of interest in AML \\ Polo-like-1 kinase inhibitors}

Polo-like kinase-1 (PLK-1) belongs to a family of serine-threonine kinases and plays an important role in centrosome maturation, spindle formation, and cytokinesis during mitosis. It is highly expressed in leukemic cells. Volasertib, a small molecule serine-threonine inhibitor, binds competitively to the kinase ATPbinding pocket and inhibits its enzymatic activity at low nanomolar concentrations. It also inhibits two related PLKs, PLK-2, and PLK-3. The encouraging data from preclinical and phase 1-2 trials led to a phase 2 randomized study of low-dose cytarabine with and without volasertib in patients with AML not suitable for frontline intensive chemotherapy. Among 87 patients randomized (median age 75 years), the addition of volasertib led to a higher overall response rate $(31 \%$ versus $13.3 \% ; p=0.052$ ) and a longer median survival (8.0 versus 5.2 months; hazard ratio $0.63 ; p=0.047)^{178}$. Unfortunately, the phase 3 pivotal trial comparing lowdose cytarabine with or without volasertib in older patients with newly diagnosed AML not eligible for intensive chemotherapy (NCT 01721876) did not meet the study endpoints. The status of volasertib is uncertain, but other presumably better PLK1 inhibitors (such as onvansertib) $)^{179}$ are under development.

\section{Antibodies targeting AML surface molecules}

Monoclonal antibodies targeting cluster designation (CD) surface molecules CD33, CD123, CD70, CLL1 (or CLEC12a), TIM3, WT1 and others, may result in important anti-AML efficacy. These antibodies may be 
unconjugated, conjugated to immunotoxins, or bispecific antibodies (BiTEs) directing killer CD3 T-cells (linking to T-cell CD3) to the AML CD surface molecules.

Unconjugated monoclonal antibodies have so far had little success in AML, as shown with CD33 unconjugated antibodies. A pilot study of azacitidine plus cusatuzumab (monoclonal unconjugated antibody-targeting CD70) was promising ${ }^{180}$. Studies of cusatuzumab combination with azacitidine and/or venetoclax are ongoing.

Monoclonal antibodies conjugated to immunotoxins have had some success, as shown by the experience with GO. Some studies with CD33 and CD123 monoclonal antibodies (e.g., SGN-33A [vadastuxumab], a humanized anti-CD33 monoclonal antibody conjugated to pyrrolobenzodiazepine) have shown excessive myelosuppression and mortality, resulting in abandoning the drug development. IMGN632 is a CD123 antibody conjugated to an alkyl-benzodiazepine. As a single-agent, IMGN632 was evaluated in 74 patients (67 AML, 7 blastic plasmadendritic cell neoplasm [BPDCN]). Among 66 evaluable patients with AML, 55\% had a reduction in bone marrow blasts, and $20 \%$ achieved a CR/CRi across a range of IMGN632 doses (0.045 to $0.3 \mathrm{mg} / \mathrm{kg}$ per course). Among seven patients with BPDCN, three (43\%) achieved a CR/ CRi. IMGN632 monotherapy is being evaluated in patients with relapsed-refractory BPDCN and MRDpositive AML. Combinations of IMGN632 with azacitidine and/or venetoclax are under evaluation in AML $(\text { NCT04086264) })^{181}$.

Ongoing studies are evaluating the delivery of radioisotopes using AML surface antigen-targeting antibodies. The clinically most advanced among these is the use of CD45-targeted antibodies (e.g., Iomab-B or 90Y-BC8DOTA $)^{182,183}$. As CD45 is ubiquitously expressed in the hematopoietic system, CD45-targeting may lead to significant myeloablation and such approaches are studied as part of pre-SCT conditioning in transplant-eligible patients. A randomized phase III study evaluating this approach with Iomab-B versus investigator choice salvage therapy prior to $\mathrm{SCT}$ in patients with relapsed-refractory AML is ongoing (NCT02665065).

The bispecific T-cell engaging antibody (BITE) technology utilizes bispecific antibody constructs that recruit CD3-effector T cells to target tumor cells (CD33, CD123, and also CD70 in the case of AML). Several AMLtargeted BiTEs are under development in AML, including flotetuzumab, AMG-330, AMG673, AMG 427, XmAb14045, AMV564. Several have shown modest activity (response rates 20 to $30 \%$ ) and were associated with the predicted toxicities (fever, hypotension, cytokine release syndrome). A potential area of research interest is exploring their efficacy in the setting of AML in CR with MRD-positive disease (as was done with blinatumomab in ALL).

\section{CAR-T cellular therapy in AML}

The success of immunotherapy in cancer led to renewed interest in developing immune-based strategies in AML, including antibody-based (discussed earlier) and cellular therapy. Trials of chimeric antigen receptor (CAR)-T cells are ongoing including autologous and allogeneic CART cells (targeting CD123, CD33, and CLL1) followed by allogeneic SCT.

\section{Summary}

Many of the hopeful predictions outlined in the AML summary of 2016 are now therapeutic realities: GO, venetoclax, FLT3 inhibitors (midostaurin, gilteritib), IDH inhibitors (ivosidenib, enasidenib), CPX-351, glasdegib, oral decitabine, and oral azacitidine. Others may soon be (quizartinib, APR246, magrolimab, menin inhibitors). The wealth of positive data allows reconsideration of what might soon be new standards of care during inductionconsolidation-SCT-maintenance in younger and older patients with AML.

\section{Acknowledgements \\ This research is supported in part by the MD Anderson Cancer Center Leukemia SPORE CA100632, the Cancer Center Support Grant (CCSG) P30CA016672, and the Charif Souki Cancer Research Grant.}

\section{Conflict of interest}

Dr. H.K. reports research grants and honoraria from AbbVie, Amgen, Ascentage BMS, Daiichi-Sankyo, Immunogen, Jazz, Novartis, Pfizer, and Sanofi; honoraria from Actinium (Advisory Board), Adaptive Biotechnologies, Aptitude Health, BioAscend, Delta Fly, Janssen Global, Oxford Biomedical, and Takeda. Dr. T.K. reports grant or research support from BMS, Celgene, Pfizer, Amgen, Jazz, AstraZeneca, and Genetech; consultant fees from Agios, Jazz, Genetech, and Novartis. Dr. C.D. reports research support to institution from Abbvie, Agios, Bayer, Calithera, Cleave, BMS/Celgene, Daiichi-Sankyo and ImmuneOnc; consultant/advisory boards with Abbvie, Agios, Celgene/BMS, Daiichi-Sankyo, ImmuneOnc, Novartis, Takeda and Notable Labs. Dr. N.D. reports research funding from Daiichi-Sankyo, Bristol-Myers Squibb, Pfizer, Gilead, Sevier, Genentech, Astellas, Daiichi-Sankyo, Abbvie, Hanmi, Trovagene, FATE, Amgen, Novimmune, Glycomimetics, and ImmunoGen and has served in a consulting or advisory role for Daiichi-Sankyo, Bristol-Myers Squibb, Pfizer, Novartis, Celgene, AbbVie, Astellas, Genentech, Immunogen, Servier, Syndax, Trillium, Gilead, Amgen, and Agios. G.B. reports research funding from Bristol-Myers Squibb, GlaxoSmithKline, Janssen Scientific Affairs, Eli Lilly and Company, Cyclacel, AstraZeneca, AbbVie, Oncoceutics, Arvinas, Cantargia, PTC

Therapeutics, Argenx, BioTheryX, and Bioline and personal fees from PTC Therapeutics, Argenx, BioTheryX, and Bioline. Dr. E.J. reports research grants and advisory rolls with AbbVie, Adaptive Biotechnologies, Amgen, BMS, Pfizer and Takeda, and advisory roll with Genetech. Dr. M.K. reports grants and other from AbbVie, Genentech, F. Hoffman La-Roche, Stemline Therapeutics, Amgen, and Forty-Seven. G.G.-M. has received grants from and acted in an advisory role for Celgene; has received grants and personal fees from Amphivena Therapeutics and Astex; and has received grants from Helsinn, Novartis, AbbVie, Onconova, H3 Biomedicine, and Merck for work performed outside of the current study. Dr. M.K. reports grants from Kisoji, Eli Lilly, Cellectis, Calithera, Ablynx, Agios, Ascentage, Astra Zeneca, other from Reata Pharmaceutical, Rafael Pharmaceutical, Sanofi. In addition, Dr. M.K. has US patents wityh Reara and Eli Lilly (US 7,795,305 B2 and a patent 62/993,166). Dr. F.R. reports research funding from BMS, Amgen, Xencor, Macrogenics, Orsenix, Abbvie, Prelude, Astex; consultancy and honoraria from Celgene, BMS, Amgen, Astellas, Xencor, Agios, AstraZeneca, and Orsenix. 


\section{Publisher's note}

Springer Nature remains neutral with regard to jurisdictional claims in published maps and institutional affiliations.

\section{Received: 21 October 2020 Revised: 14 December 2020 Accepted: 18 January 2021 \\ Published online: 22 February 2021}

\section{References}

1. Kantarjian, H. Acute myeloid leukemia-major progress over four decades and glimpses into the future. Am. J. Hematol. 91, 131-145 (2016).

2. Short, N. J., Rytting, M. E. \& Cortes, J. E. Acute myeloid leukaemia. Lancet $\mathbf{3 9 2}$ 593-606 (2018).

3. Kadia, T. M., Ravandi, F., Cortes, J. \& Kantarjian, H. Toward individualized therapy in acute myeloid leukemia: a contemporary review. JAMA Oncol. 1 820-828 (2015).

4. Kadia, T. M., Ravandi, F., O'Brien, S., Cortes, J. \& Kantarjian, H. M. Progress in acute myeloid leukemia. Clin. Lymphoma Myeloma Leuk. 15, 139-151 (2015).

5. Dohner, H., Weisdorf, D. J. \& Bloomfield, C. D. Acute myeloid leukemia. N. Engl. J. Med. 373, 1136-1152 (2015).

6. Lowenberg, B. et al. High-dose daunorubicin in older patients with acute myeloid leukemia. N. Engl. J. Med. 361, 1235-1248 (2009).

7. Fernandez, H. F. et al. Anthracycline dose intensification in acute myeloid leukemia. N. Engl. J. Med. 361, 1249-1259 (2009).

8. Ravandi, F. et al. Effective treatment of acute promyelocytic leukemia with alltrans-retinoic acid, arsenic trioxide, and gemtuzumab ozogamicin. J. Clin. Oncol. 27, 504-510 (2009).

9. Lo-Coco, F. et al. Retinoic acid and arsenic trioxide for acute promyelocytic leukemia. N. Engl. J. Med. 369, 111-121 (2013).

10. Abaza, Y. et al. Long-term outcome of acute promyelocytic leukemia treated with all-trans-retinoic acid, arsenic trioxide, and gemtuzumab. Blood 129 1275-1283 (2017).

11. Platzbecker, U. et al. Improved outcomes with retinoic acid and arsenic trioxide compared with retinoic acid and chemotherapy in non-high-risk acute promyelocytic leukemia: final results of the randomized Italian-German APL0406 trial. J. Clin. Oncol. 35, 605-612 (2017)

12. Burnett, A. K. et al. Arsenic trioxide and all-trans retinoic acid treatment for acute promyelocytic leukaemia in all risk groups (AML17): results of a randomised, controlled, phase 3 trial. Lancet Oncol. 16, 1295-1305 (2015).

13. Borthakur, G. et al. Gemtuzumab ozogamicin with fludarabine, cytarabine and granulocyte colony stimulating factor ( $F L A G-G O)$ as front-line regimen in patients with core binding factor acute myelogenous leukemia. Am. J. Hematol. 89, 964-968 (2014).

14. Borthakur, G., Cortes, J. \& Ravandi, F. Fludarabine, cytarabine, G-CSF and gemtuzumab ozogamicin (FLAG-GO) regimen results in better molecular response and relapse-free survival in core binding factor acute myeloid leukemia than FLAG and idarubicin (FLAG-Ida). Blood 134, 290 (2019).

15. Burnett, A. K. et al. Identification of patients with acute myeloblastic leukemia who benefit from the addition of gemtuzumab ozogamicin: results of the MRC AML15 trial. J. Clin. Oncol. 29, 369-377 (2011).

16. Petersdorf, S. H. et al. A phase 3 study of gemtuzumab ozogamicin during induction and postconsolidation therapy in younger patients with acute myeloid leukemia. Blood 121, 4854-4860 (2013).

17. Hills, R. K. et al. Addition of gemtuzumab ozogamicin to induction chemotherapy in adult patients with acute myeloid leukaemia: a meta-analysis of individual patient data from randomised controlled trials. Lancet Oncol. 15, 986-996 (2014).

18. Patel, J. P. et al. Prognostic relevance of integrated genetic profiling in acute myeloid leukemia. N. Engl. J. Med. 366, 1079-1089 (2012).

19. Cancer Genome Atlas Research Network. et al. Genomic and epigenomic landscapes of adult de novo acute myeloid leukemia. N. Engl. J. Med. 368 2059-2074 (2013).

20. Ding, $L$ et al. Clonal evolution in relapsed acute myeloid leukaemia revealed by whole-genome sequencing. Nature 481, 506-510 (2012).

21. Papaemmanuil, E. et al. Genomic classification and prognosis in acute myeloid leukemia. N. Engl. J. Med. 374, 2209-2221 (2016).

22. Grimwade, D. et al. Refinement of cytogenetic classification in acute myeloid leukemia: determination of prognostic significance of rare recurring chromosomal abnormalities among 5876 younger adult patients treated in the United Kingdom Medical Research Council trials. Blood 116, 354-365 (2010)

23. Pastore, F. et al. Combined molecular and clinical prognostic index for relapse and survival in cytogenetically normal acute myeloid leukemia. J. Clin. Oncol. 32, 1586-1594 (2014).

24. Richard-Carpentier, G. \& DiNardo, C. D. Single-agent and combination biologics in acute myeloid leukemia. Hematol. Am. Soc. Hematol. Educ. Program 2019, 548-556 (2019).

25. Short, N. J. et al. Advances in the treatment of acute myeloid leukemia: new drugs and new challenges. Cancer Discov. 10, 506-525 (2020).

26. Kantarjian, H. M. et al. Multicenter, randomized, open-label, phase III trial of decitabine versus patient choice, with physician advice, of either supportive care or low-dose cytarabine for the treatment of older patients with newly diagnosed acute myeloid leukemia. J. Clin. Oncol. 30, 2670-2677 (2012).

27. Tuma, R. Withdrawl will have major impact on patients, some leukemia experts say. Oncol. Times 22, 11-13 (2010).

28. Dombret, $\mathrm{H}$. et al. International phase 3 study of azacitidine vs conventional care regimens in older patients with newly diagnosed AML with $>30 \%$ blasts. Blood 126, 291-299 (2015).

29. Ravandi, F. et al. Vosaroxin plus cytarabine versus placebo plus cytarabine in patients with first relapsed or refractory acute myeloid leukaemia (VALOR): a randomised, controlled, double-blind, multinational, phase 3 study. Lancet Oncol. 16, 1025-1036 (2015).

30. Dohner, $H$. et al. Diagnosis and management of AML in adults: 2017 ELN recommendations from an international expert panel. Blood 129, 424-447 (2017).

31. Tallman, M. S. et al. Acute Myeloid Leukemia, Version 3.2019, NCCN Clinical Practice Guidelines in Oncology. J. Natl. Compr. Canc. Netw. 17, 721-749 (2019).

32. DiNardo, C. D. et al. Characteristics, clinical outcome, and prognostic significance of IDH mutations in AML. Am. J. Hematol. 90, 732-736 (2015).

33. Santos, F. P. et al. Prognostic value of FLT3 mutations among different cytogenetic subgroups in acute myeloid leukemia. Cancer 117, 2145-2155 (2011).

34. Pratcorona, M. et al. Favorable outcome of patients with acute myeloid leukemia harboring a low-allelic burden FLT3-ITD mutation and concomitant NPM1 mutation: relevance to post-remission therapy. Blood 121, 2734-2738 (2013).

35. Schlenk, R. F. et al. Differential impact of allelic ratio and insertion site in FLT3ITD-positive AML with respect to allogeneic transplantation. Blood $\mathbf{1 2 4}$ 3441-3449 (2014)

36. Ho, A. D. et al. Allogeneic stem cell transplantation improves survival in patients with acute myeloid leukemia characterized by a high allelic ratio of mutant FLT3-ITD. Biol. Blood Marrow Transplant 22, 462-469 (2016).

37. Schlenk, R. F. et al. Mutations and treatment outcome in cytogenetically normal acute myeloid leukemia. N. Engl. J. Med. 358, 1909-1918 (2008).

38. Seifert, $H$. et al. The prognostic impact of $17 p$ (p53) deletion in 2272 adults with acute myeloid leukemia. Leukemia 23, 656-663 (2009).

39. Kadia, T. M. et al. TP53 mutations in newly diagnosed acute myeloid leukemia: Clinicomolecular characteristics, response to therapy, and outcomes. Cancer 122, 3484-3491 (2016).

40. Boddu, P. et al. Outcomes with lower intensity therapy in TP53-mutated acute myeloid leukemia. Leuk. Lymphoma 59, 2238-2241 (2018).

41. Sasaki, $K$. et al. Impact of the variant allele frequency of ASXL1, DNMT3A JAK2, TET2, TP53, and NPM1 on the outcomes of patients with newly diagnosed acute myeloid leukemia. Cancer 126, 765-774 (2020).

42. Thol, F. et al. Incidence and prognostic influence of DNMT3A mutations in acute myeloid leukemia. J. Clin. Oncol. 29, 2889-2896 (2011).

43. Ley, T. J. et al. DNMT3A mutations in acute myeloid leukemia. N. Engl. J. Med. 363, 2424-2433 (2010).

44. Mendler, J. H. et al. RUNX1 mutations are associated with poor outcome in younger and older patients with cytogenetically normal acute myeloid leukemia and with distinct gene and MicroRNA expression signatures. J. Clin. Oncol. 30, 3109-3118 (2012).

45. Gaidzik, V. I. et al. RUNX1 mutations in acute myeloid leukemia: results from a comprehensive genetic and clinical analysis from the AML study group. J. Clin. Oncol. 29, 1364-1372 (2011).

46. Groschel, S. et al. Deregulated expression of EVI1 defines a poor prognostic subset of MLL-rearranged acute myeloid leukemias: a study of the German- 
Austrian Acute Myeloid Leukemia Study Group and the Dutch-Belgian-Swiss HOVON/SAKK Cooperative Group. J. Clin. Oncol. 31, 95-103 (2013).

47. Cairoli, R. et al. Prognostic impact of C-KIT mutations in core binding factor leukemias: an Italian retrospective study. Blood 107, 3463-3468 (2006).

48. Paschka, P. et al. Adverse prognostic significance of KIT mutations in adult acute myeloid leukemia with inv(16) and $\mathrm{t}(8 ; 21)$ : a Cancer and Leukemia Group B Study. J. Clin. Oncol. 24, 3904-3911 (2006).

49. Schnittger, S. et al. KIT-D816 mutations in AML1-ETO-positive AML are associated with impaired event-free and overall survival. Blood 107, 1791-1799 (2006).

50. Marcucci, G. et al. Adding the KIT inhibitor dasatinib (DAS) to standard induction and consolidation therapy for newly diagnosed patients (pts) with core binding factor (CBF) acute myeloid leukemia (AML): initial results of the CALGB 10801 (Alliance) Study. Blood 124, 8, https:/doi.org/10.1182/blood. V124.21.8.8 (2014).

51. Gotlib, J. et al. Avapritinib induces responses in patients (PTS) with advanced systemic mastocytosis (ADVSM), regardless of prior midostaurin therapy. EHA Abstract EP1079, (2020)

52. Angenendt, L. et al. Chromosomal abnormalities and prognosis in NPM1mutated acute myeloid leukemia: a pooled analysis of individual patient data from nine international cohorts. J. Clin. Oncol. 37, 2632-2642 (2019).

53. Chan, S. M. et al. Isocitrate dehydrogenase 1 and 2 mutations induce BCL-2 dependence in acute myeloid leukemia. Nat. Med. 21, 178-184 (2015).

54. Welch, J. S. et al. TP53 and decitabine in acute myeloid leukemia and myelodysplastic syndromes. N. Engl. J. Med. 375, 2023-2036 (2016).

55. Klossowski, S. et al. Menin inhibitor Ml-3454 induces remission in MLL1rearranged and NPM1-mutated models of leukemia. J. Clin. Invest. 130 981-997 (2020).

56. Jongen-Lavrencic, M. et al. Molecular minimal residual disease in acute myeloid leukemia. N. Engl. J. Med. 378, 1189-1199 (2018).

57. Grimwade, D. \& Freeman, S. D. Defining minimal residual disease in acute myeloid leukemia: which platforms are ready for "prime time"? Blood 124 3345-3355 (2014).

58. Pastore, F. \& Levine, R. L. Next-Generation sequencing and detection of minimal residual disease in acute myeloid leukemia: ready for clinical practice? JAMA 314, 778-780 (2015).

59. Klco, J. M. et al. Association between mutation clearance after induction therapy and outcomes in acute myeloid leukemia. JAMA 314, 811-822 (2015).

60. Ravandi, F. et al. Persistence of minimal residual disease assessed by multiparameter flow cytometry is highly prognostic in younger patients with acute myeloid leukemia. Cancer 123, 426-435 (2017).

61. Short, N. J. et al. Association of measurable residual disease with survival outcomes in patients with acute myeloid leukemia: a systematic review and meta-analysis. JAMA Oncol. 6, 1890-1899 (2020).

62. Hourigan, C. S. et al. Impact of conditioning intensity of allogeneic transplantation for acute myeloid leukemia with genomic evidence of residual disease. J. Clin. Oncol. 38, 1273-1283 (2020).

63. Grimwade, D. et al. Prospective minimal residual disease monitoring to predict relapse of acute promyelocytic leukemia and to direct pre-emptive arsenic trioxide therapy. J. Clin. Oncol. 27, 3650-3658 (2009).

64. Yin, J. A. et al. Minimal residual disease monitoring by quantitative RT-PCR in core binding factor AML allows risk stratification and predicts relapse: results of the United Kingdom MRC AML-15 trial. Blood 120, 2826-2835 (2012).

65. Zhu, H. H. et al. MRD-directed risk stratification treatment may improve outcomes of $t(8 ; 21) A M L$ in the first complete remission: results from the AML05 multicenter trial. Blood 121, 4056-4062 (2013).

66. Kronke, J. et al. Monitoring of minimal residual disease in NPM1-mutated acute myeloid leukemia: a study from the German-Austrian acute myeloid leukemia study group. J. Clin. Oncol. 29, 2709-2716 (2011).

67. Schnittger, S. et al. Minimal residual disease levels assessed by NPM1 mutation-specific RQ-PCR provide important prognostic information in AML. Blood 114, 2220-2231 (2009).

68. Kantarjian, H. M. et al. Acute promyelocytic leukemia. M.D. Anderson Hospital experience. Am. J. Med. 80, 789-797 (1986).

69. Kantarjian, H. M. et al. Role of maintenance chemotherapy in acute promyelocytic leukemia. Cancer 59, 1258-1263 (1987).

70. Lo-Coco, F. et al. Gemtuzumab ozogamicin (Mylotarg) as a single agent for molecularly relapsed acute promyelocytic leukemia. Blood 104, 1995-1999 (2004)

71. Soignet, S. L. et al. United States multicenter study of arsenic trioxide in relapsed acute promyelocytic leukemia. J. Clin. Oncol. 19, 3852-3860 (2001).
72. Fenaux, P. et al. A randomized comparison of all transretinoic acid (ATRA) followed by chemotherapy and ATRA plus chemotherapy and the role of maintenance therapy in newly diagnosed acute promyelocytic leukemia. The European APL Group. Blood 94, 1192-1200 (1999).

73. Sanz, M. A. et al. A modified AIDA protocol with anthracycline-based consolidation results in high antileukemic efficacy and reduced toxicity in newly diagnosed PML/RARalpha-positive acute promyelocytic leukemia. PETHEMA group. Blood 94, 3015-3021 (1999).

74. Tallman, M. S. et al. All-trans retinoic acid in acute promyelocytic leukemia: long-term outcome and prognostic factor analysis from the North American Intergroup protocol. Blood 100, 4298-4302 (2002).

75. Powell, B. L. et al. Arsenic trioxide improves event-free and overall survival for adults with acute promyelocytic leukemia: North American Leukemia Intergroup Study C9710. Blood 116, 3751-3757 (2010).

76. Avvisati, G. et al. AIDA 0493 protocol for newly diagnosed acute promyelocytic leukemia: very long-term results and role of maintenance. Blood 117, 4716-4725 (2011).

77. Russell, N. et al. Attenuated arsenic trioxide plus ATRA therapy for newly diagnosed and relapsed APL: long-term follow-up of the AML17 trial. Blood 132, 1452-1454 (2018).

78. Lancet, J. E. et al. A phase 2 study of ATRA, arsenic trioxide, and gemtuzumab ozogamicin in patients with high-risk APL (SWOG 0535). Blood Adv. 4 1683-1689 (2020).

79. Tsimberidou, A. M. et al. Granulocyte colony stimulating factor administration associated with cerebral hemorrhage in acute promyelocytic leukemia. Leukemia 20, 1452-1453 (2006).

80. Chamoun, K. et al. Unrecognized fluid overload during induction therapy increases morbidity in patients with acute promyelocytic leukemia. Cancer 125, 3219-3224 (2019).

81. Ravandi, F. et al. Oral arsenic trioxide ORH-2014 pharmacokinetic and safety profile in patients with advanced hematologic disorders. Haematologica 105, 1567-1574 (2021).

82. $\mathrm{Zhu}, \mathrm{H}$. H. et al. Oral arsenic plus retinoic acid versus intravenous arsenic plus retinoic acid for non-high-risk acute promyelocytic leukaemia: a non-inferiority, randomised phase 3 trial. Lancet Oncol. 19, 871-879 (2018).

83. Byrd, J. C. et al. Repetitive cycles of high-dose cytarabine benefit patients with acute myeloid leukemia and inv(16)(p13q22) or t(16;16)(p13;q22): results from CALGB 8461. J. Clin. Oncol. 22, 1087-1094 (2004).

84. Paschka, P. et al. Secondary genetic lesions in acute myeloid leukemia with inv(16) or t(16;16): a study of the German-Austrian AML Study Group (AMLSG). Blood 121, 170-177 (2013).

85. Sasaki, K. et al. De novo acute myeloid leukemia: a population- based study of outcome in the united states based on the surveillance, epidemiology, and end results (SEER) database, 1980-2017. Cancer (in press) (2021).

86. Mayer, R. J. et al. Intensive postremission chemotherapy in adults with acute myeloid leukemia. Cancer and Leukemia Group B. N. Engl. J. Med. 331 896-903 (1994).

87. Burnett, A. K. et al. Optimization of chemotherapy for younger patients with acute myeloid leukemia: results of the medical research council AML15 trial. J. Clin. Oncol. 31, 3360-3368 (2013).

88. Weick, J. K. et al. A randomized investigation of high-dose versus standarddose cytosine arabinoside with daunorubicin in patients with previously untreated acute myeloid leukemia: a Southwest Oncology Group study. Blood 88, 2841-2851 (1996).

89. Bishop, J. F. et al. A randomized study of high-dose cytarabine in induction in acute myeloid leukemia. Blood 87, 1710-1717 (1996).

90. Kern, W. \& Estey, E. H. High-dose cytosine arabinoside in the treatment of acute myeloid leukemia: Review of three randomized trials. Cancer 107, 116-124 (2006).

91. Lowenberg, B. et al. Cytarabine dose for acute myeloid leukemia. N. Engl. J. Med. 364, 1027-1036 (2011).

92. Willemze, R. et al. High-dose cytarabine in induction treatment improves the outcome of adult patients younger than age 46 years with acute myeloid leukemia: results of the EORTC-GIMEMA AML-12 trial. J. Clin. Oncol. 32, 219-228 (2014).

93. Bassan, R. et al. Randomized trial comparing standard vs sequential highdose chemotherapy for inducing early CR in adult AML. Blood Adv. 3, 1103-1117 (2019).

94. Garcia-Manero, G. et al. Standard versus high-dose cytarabine with or without vorinostat in acute myelogenous leukemia: results of SWOG 1203. Leukemia (in press), (2021). 
95. Plunkett, W. et al. Saturation of 1-beta-D-arabinofuranosylcytosine 5'-triphosphate accumulation in leukemia cells during high-dose 1-beta-Darabinofuranosylcytosine therapy. Cancer Res. 47, 3005-3011 (1987).

96. Plunkett, W., Liliemark, J. O., Estey, E. \& Keating, M. J. Saturation of ara-CTP accumulation during high-dose ara- $C$ therapy: pharmacologic rationale for intermediate-dose ara-C. Semin. Oncol. 14, 159-166 (1987).

97. Estey, E. H. et al. Randomized phase II study of fludarabine + cytosine arabinoside + idarubicin +/- granulocyte colony-stimulating factor in poor prognosis newly diagnosed acute myeloid leukemia and myelodysplastic syndrome. Blood 93, 2478-2484 (1999).

98. Burnett, A. K. et al. Addition of gemtuzumab ozogamicin to induction chemotherapy improves survival in older patients with acute myeloid leukemia. J. Clin. Oncol. 30, 3924-3931 (2012).

99. Park, Y. et al. High-dose cytarabine consolidation $(\geq 1.5 \mathrm{~g} / \mathrm{m} 2)$ might have shown a better outcomes than intermediate-dose cytarabine $(1.0 \mathrm{~g} / \mathrm{m} 2)$ combined with anthracyclines in AML patients who had achieved complete remissions in the first induction by standard $3+7$ regimen. Blood 122, 2692 (2013).

100. Holowiecki, J. et al. Addition of cladribine to daunorubicin and cytarabine increases complete remission rate after a single course of induction treatment in acute myeloid leukemia. Multicenter, phase III study. Leukemia $\mathbf{1 8}$ 989-997 (2004)

101. Holowiecki, J. et al. Cladribine, but not fludarabine, added to daunorubicin and cytarabine during induction prolongs survival of patients with acute myeloid leukemia: a multicenter, randomized phase III study. J. Clin. Oncol. 30 2441-2448 (2012).

102. Jabbour, E. et al. A randomized phase 2 study of idarubicin and cytarabine with clofarabine or fludarabine in patients with newly diagnosed acute myeloid leukemia. Cancer 123, 4430-4439 (2017).

103. Devillier, R., Bertoli, S. \& Prebet, T. Induction therapy for AML patients with daunorubicin dose of $60 \mathrm{mg} / \mathrm{m}^{2}$ and $90 \mathrm{mg} / \mathrm{m}^{2}$ results in similar complete response rate, relapse-free and overall survival. Blood 122, 66 (2013).

104. Burnett, A. K. et al. A randomized comparison of daunorubicin $90 \mathrm{mg} / \mathrm{m} 2 \mathrm{vs}$ $60 \mathrm{mg} / \mathrm{m} 2$ in AML induction: results from the UK NCRI AML17 trial in 1206 patients. Blood 125, 3878-3885 (2015).

105. A systematic collaborative overview of randomized trials comparing idarubicin with daunorubicin (or other anthracyclines) as induction therapy for acute myeloid leukaemia. AML Collaborative Group. Br. J. Haematol. 103 100-109 (1998).

106. Pautas, C. et al. Randomized study of intensified anthracycline doses for induction and recombinant interleukin-2 for maintenance in patients with acute myeloid leukemia age 50 to 70 years: results of the ALFA-9801 study. J. Clin. Oncol. 28, 808-814 (2010).

107. Gardin, C. et al. Superior long-term outcome with idarubicin compared with high-dose daunorubicin in patients with acute myeloid leukemia age 50 years and older. J. Clin. Oncol. 31, 321-327 (2013).

108. Mandelli, F. et al. Daunorubicin versus mitoxantrone versus idarubicin as induction and consolidation chemotherapy for adults with acute myeloid leukemia: the EORTC and GIMEMA Groups Study AML-10. J. Clin. Oncol. 27, 5397-5403 (2009).

109. Bross, P. F. et al. Approval summary: gemtuzumab ozogamicin in relapsed acute myeloid leukemia. Clin. Cancer Res. 7, 1490-1496 (2001).

110. Castaigne, S. et al. Effect of gemtuzumab ozogamicin on survival of adult patients with de-novo acute myeloid leukaemia (ALFA-0701): a randomised, open-label, phase 3 study. Lancet 379, 1508-1516 (2012).

111. Delaunay, J. et al. Addition of gemtuzumab ozogamycin to chemotherapy improves event-free survival but not overall survival of AML patients with intermediate cytogenetics not eligible for allogeneic transplantation. Results of the GOELAMS AML 2006 IR Study. Blood 118, 79 (2011).

112. Kharfan-Dabaja, M. A. A new dawn for gemtuzumab ozogamicin? Lancet Oncol. 15, 913-914 (2014).

113. Ravandi, F. et al. Gemtuzumab ozogamicin: time to resurrect? J. Clin. Oncol. 30, 3921-3923 (2012)

114. Pigneux, A. et al. Addition of lomustine to idarubicin and cytarabine improves the outcome of elderly patients with de novo acute myeloid leukemia: a report from the GOELAMS. J. Clin. Oncol. 28, 3028-3034 (2010)

115. Lachowiez, C. et al. Interim analysis of the Phase $1 \mathrm{~b} / 2$ study of the $B C L-2$ inhibitor venetoclax in combination with standard intensive AML induction/ consolidation therapy with FLAG-IDA in patients with newly diagnosed or relapsed/refractory AML. Blood 136, 332 (2020).
116. Reville, P. K., Kantarjian, H. \& Borthakur, G. Cladribine, idarubicin, cytarabine (ara-C), and venetoclax in treating patients with acute myeloid leukemia and high-risk myelodysplastic syndrome. Blood 136, 2854 (2020).

117. Halpern, A. B., Lyman, G. H., Walsh, T. J., Kontoyiannis, D. P. \& Walter, R. B. Primary antifungal prophylaxis during curative-intent therapy for acute myeloid leukemia. Blood 126, 2790-2797 (2015).

118. Cornely, O. A. et al. Posaconazole vs. fluconazole or itraconazole prophylaxis in patients with neutropenia. N. Engl. J. Med. 356, 348-359 (2007).

119. Stein, E. M. et al. Ivosidenib or enasidenib combined with intensive chemotherapy in patients with newly diagnosed AML: a phase 1 study. Blood 2020007233, https://doi.org/10.1182/blood.2020007233. (2020). Epub ahead of print.

120. Stone, R. M. et al. Midostaurin plus chemotherapy for acute myeloid leukemia with a FLT3 mutation. N. Engl. J. Med. 377, 454-464 (2017).

121. Alfayez, $M$. et al. Outcomes with subsequent FLT3-inhibitor (FLT3i) based therapies in FLT3-mutated (mu) patients (pts) refractory/relapsed (R/R) to one or more prior FLT3 inhibitor based therapies: a single center experience. Blood 132, 633 (2018).

122. Pratz, K. W. et al. A Phase 1 Study of Gilteritinib in Combination with Induction and Consolidation Chemotherapy in Patients with Newly Diagnosed AML: Final Results. Blood 136, abst 24 (2020).

123. Xuan, L. et al. Sorafenib maintenance in patients with FLT3-ITD acute myeloid leukaemia undergoing allogeneic haematopoietic stem-cell transplantation: an open-label, multicentre, randomised phase 3 trial. Lancet Oncol. 21, 1201-1212 (2020).

124. Burchert, A. et al. Sorafenib maintenance after allogeneic hematopoietic stem cell transplantation for acute myeloid leukemia With FLT3-internal tandem duplication mutation (SORMAIN). J. Clin. Oncol. 38, 2993-3002 (2020).

125. Libura, M. et al. Cladribine added to daunorubicin-cytarabine induction prolongs survival of FLT3-ITD + normal karyotype AML patients. Blood 127 360-362 (2016).

126. Choi, E. J. et al. Comparison of anthracyclines used for induction chemotherapy in patients with FLT3-ITD-mutated acute myeloid leukemia. Leuk. Res. 68, 51-56 (2018).

127. Kantarjian, H. et al. Results of intensive chemotherapy in 998 patients age 65 years or older with acute myeloid leukemia or high-risk myelodysplastic syndrome: predictive prognostic models for outcome. Cancer 106, 1090-1098 (2006).

128. Kantarjian, $\mathrm{H}$. et al. Intensive chemotherapy does not benefit most older patients (age 70 years or older) with acute myeloid leukemia. Blood $\mathbf{1 1 6}$ 4422-4429 (2010).

129. Quintas-Cardama, A. et al. Epigenetic therapy is associated with similar survival compared with intensive chemotherapy in older patients with newly diagnosed acute myeloid leukemia. Blood 120, 4840-4845 (2012).

130. Takahashi, K. et al. Clofarabine plus low-dose cytarabine is as effective as and less toxic than intensive chemotherapy in elderly AML patients. Clin. Lymphoma Myeloma Leuk. 16, 163-8 e1-2 (2016).

131. Bitterman, R. et al. Baseline chest computed tomography for early diagnosis of invasive pulmonary aspergillosis in hemato-oncological patients: a prospective cohort study. Clin. Infect. Dis. 69, 1805-1808 (2019).

132. Lang, K. et al. Trends in the treatment of acute myeloid leukaemia in the elderly. Drugs Aging 22, 943-955 (2005).

133. Burnett, A. K et al. A comparison of low-dose cytarabine and hydroxyurea with or without all-trans retinoic acid for acute myeloid leukemia and highrisk myelodysplastic syndrome in patients not considered fit for intensive treatment. Cancer 109, 1114-1124 (2007).

134. Blum, W. et al. Clinical response and miR-29b predictive significance in older AML patients treated with a 10-day schedule of decitabine. Proc. Natl Acad. Sci. USA 107, 7473-7478 (2010)

135. Savona, M. R. et al. An oral fixed-dose combination of decitabine and cedazuridine in myelodysplastic syndromes: a multicentre, open-label, doseescalation, phase 1 study. Lancet Haematol. 6, e194-e203 (2019).

136. Garcia-Manero, G. et al. Oral cedazuridine/decitabine for MDS and CMML: a phase 2 pharmacokinetic/pharmacodynamic randomized crossover study. Blood 136, 674-683 (2020).

137. Kadia, T. M. et al. Cladribine and low-dose cytarabine alternating with decitabine as front-line therapy for elderly patients with acute myeloid leukaemia: a phase 2 single-arm trial. Lancet Haematol. 5, e411-e421 (2018).

138. Kadia, T. M. et al. Final results of a phase 2 trial of clofarabine and low-dose cytarabine alternating with decitabine in older patients with newly diagnosed acute myeloid leukemia. Cancer 121, 2375-2382 (2015). 
139. Pan, R. et al. Selective BCL-2 inhibition by ABT-199 causes on-target cell death in acute myeloid leukemia. Cancer Discov. 4, 362-375 (2014).

140. Konopleva, M. et al. Efficacy and biological correlates of response in a phase II study of venetoclax monotherapy in patients with acute myelogenous leukemia. Cancer Discov. 6, 1106-1117 (2016).

141. Wei, A. H. et al. Venetoclax combined with low-dose cytarabine for previously untreated patients with acute myeloid leukemia: results from a phase Ib/ll study. J. Clin. Oncol. 37, 1277-1284 (2019).

142. DiNardo, C. D. et al. Venetoclax combined with decitabine or azacitidine in treatment-naive, elderly patients with acute myeloid leukemia. Blood 133, 7-17 (2019)

143. DiNardo, C. D. et al. Azacitidine and venetoclax in previously untreated acute myeloid leukemia. N. Engl. J. Med. 383, 617-629 (2020).

144. Wei, A. H. et al. Venetoclax plus LDAC for newly diagnosed AML ineligible for intensive chemotherapy: a phase 3 randomized placebo-controlled trial. Blood 135, 2137-2145 (2020).

145. DiNardo, C. D. et al. 10-day decitabine with venetoclax for newly diagnosed intensive chemotherapy ineligible, and relapsed or refractory acute myeloid leukaemia: a single-centre, phase 2 trial. Lancet Haematol. 7, e724-e736 (2020)

146. Kadia, T. et al. Phase II study of venetoclax added to cladribine + low dose AraC (LDAC) alternating with 5-azacytidine demonstrates high rates of minimal residual disease (MRD) negative complete remissions (CR) and excellent tolerability in older patients with newly diagnosed acute myeloid leukemia (AML). Blood 136, 25 (2020).

147. Ohanian, M. et al. Sorafenib combined with 5-azacytidine in older patients with untreated FLT3-ITD mutated acute myeloid leukemia. Am. J. Hematol. 93, 1136-1141 (2018).

148. Esteve, J. et al. Multicenter, open-label, 3-arm study of gilteritinib, gilteritinib plus azacitidine, or azacitidine alone in newly diagnosed FLT3 mutated (FLT3mut+) acute myeloid leukemia (AML) patients ineligible for intensive induction chemotherapy: findings from the safety cohort. Blood 132(Supplement 1), 2736, https://doi.org/10.1182/blood-2018-99-110976 (2018).

149. Feldman, E. J. et al. First-in-man study of CPX-351: a liposomal carrier containing cytarabine and daunorubicin in a fixed 5:1 molar ratio for the treatment of relapsed and refractory acute myeloid leukemia. J. Clin. Oncol. 29, 979-985 (2011)

150. Lancet, J. E. et al. Phase 2 trial of CPX-351, a fixed 5:1 molar ratio of cytarabine/daunorubicin, vs cytarabine/daunorubicin in older adults with untreated AML. Blood 123, 3239-3246 (2014).

151. Lancet, J. E. et al. CPX-351 (cytarabine and daunorubicin) liposome for injection versus conventional cytarabine plus daunorubicin in older patients with newly diagnosed secondary acute myeloid leukemia. J. Clin. Oncol. 36, 2684-2692 (2018).

152. Jamieson, C., Martinelli, G., Papayannidis, C. \& Cortes, J. E. Hedgehog pathway inhibitors: a new therapeutic class for the treatment of acute myeloid leukemia. Blood Cancer Discov. 1, 134-145 (2020).

153. Cortes, J. E. et al. Randomized comparison of low dose cytarabine with or without glasdegib in patients with newly diagnosed acute myeloid leukemia or high-risk myelodysplastic syndrome. Leukemia 33, 379-389 (2019).

154. Norsworthy, K. J. et al. FDA Approval summary: glasdegib for newly diagnosed acute myeloid leukemia. Clin. Cancer Res. 25, 6021-6025 (2019).

155. Lehmann, S. et al. Targeting p53 in vivo: a first-in-human study with p53targeting compound APR-246 in refractory hematologic malignancies and prostate cancer. J. Clin. Oncol. 30, 3633-3639 (2012).

156. Sallman, D. A. et al. Phase 2 results of APR-246 and azacitidine (AZA) in patients with TP53 mutant myelodysplastic syndromes (MDS) and oligoblastic acute myeloid leukemia (AML). Blood 134, 676 (2019).

157. Cluzeau, T. et al. APR-246 combined with azacitidine in TP53 mutated myelodysplastic syndromes (MDS) and acute myeloid leukemia. A phase 2 study by the Groupe FrancoPhone des Myelodysplasies (GFM) A. Blood 134, 677 (2020).

158. Jaiswal, S. et al. CD47 is upregulated on circulating hematopoietic stem cells and leukemia cells to avoid phagocytosis. Cell 138, 271-285 (2009).

159. Majeti, R. et al. CD47 is an adverse prognostic factor and therapeutic antibody target on human acute myeloid leukemia stem cells. Cell 138, 286-299 (2009).

160. Advani, R. et al. CD47 blockade by Hu5F9-G4 and rituximab in non-hodgkin's lymphoma. N. Engl. J. Med. 379, 1711-1721 (2018).
161. Sallman, D. A. et al. The first-in-class anti-CD47 antibody magrolimab in combination with azacitidine is effective in AML patients: phase $1 \mathrm{~b}$ results. Blood 134, 330 (2020).

162. Wei, A. et al. Maintenance Therapy with CC-486 for acute myeloid leukemia in first remission. N. Engl. J. Med. 383, 2526-2537, https://doi.org/10.1056/ NEJMoa2004444 (2020)

163. Huls, G. et al. Azacitidine maintenance after intensive chemotherapy improves DFS in older AML patients. Blood 133, 1457-1464 (2019).

164. Perl, A. E. et al. Gilteritinib or chemotherapy for relapsed or refractory FLT3mutated AML. N. Engl. J. Med. 381, 1728-1740 (2019).

165. Derolf, A. et al. Decreasing early mortality in acute myeloid leukaemia in Sweden 1997-2014: improving performance status is a major contributing factor. Br. J. Haematol. 188, 187-191 (2020).

166. Bhatt, V. R. et al. Early mortality and overall survival of acute myeloid leukemia based on facility type. Am. J. Hematol. 92, 764-771 (2017)

167. Ho, G. et al. Decreased early mortality associated with the treatment of acute myeloid leukemia at National Cancer Institute-designated cancer centers in California. Cancer 124, 1938-1945 (2018).

168. Koreth, J. et al. Allogeneic stem cell transplantation for acute myeloid leukemia in first complete remission: systematic review and meta-analysis of prospective clinical trials. JAMA 301, 2349-2361 (2009).

169. Burnett, A. K. et al. The value of allogeneic bone marrow transplant in patients with acute myeloid leukaemia at differing risk of relapse: results of the UK MRC AML 10 trial. Br. J. Haematol. 118, 385-400 (2002).

170. Jabbour, E. et al. Twice-daily fludarabine and cytarabine combination with or without gentuzumab ozogamicin is effective in patients with relapsed/ refractory acute myeloid leukemia, high-risk myelodysplastic syndrome, and blast- phase chronic myeloid leukemia. Clin. Lymphoma Myeloma Leuk. 12, 244-251 (2012).

171. Perl, A. E. et al. Selective inhibition of FLT3 by gilteritinib in relapsed or refractory acute myeloid leukaemia: a multicentre, first-in-human, open-label, phase 1-2 study. Lancet Oncol. 18, 1061-1075 (2017).

172. Daver, N. et al. Efficacy and safety of venetoclax in combination with gilteritinib for relapsed/refractory FLT3-mutated acute myeloid leukemia in the expansion cohort of a phase 1b study. Blood 134, 333 (2020). (Abstract).

173. Amatangelo, M. D. et al. Enasidenib induces acute myeloid leukemia cell differentiation to promote clinical response. Blood 130, 732-741 (2017).

174. Stein, E. M. et al. Safety and efficacy of AG-221, a potent inhibitor of mutant IDH2 that promotes differentiation of myeloid cells in patients with advanced hematologic malignancies: results of a phase 1/2 trial. Blood 126, 323 (2015)

175. DiNardo, C. D. et al. Enasidenib plus azacitidine significantly improves complete remission and overall response compared with azacitidine alone in patients with newly diagnosed acute myeloid leukemia (AML) with isocitrate dehydrogenase 2 (IDH2) mutations: interim phase II results from an ongoing, randomized study. Blood 134, 643 (2019).

176. DiNardo, C. D. et al. Durable remissions with ivosidenib in IDH1-mutated relapsed or refractory AML. N. Engl. J. Med. 378, 2386-2398 (2018).

177. Choe, S. et al. Molecular mechanisms mediating relapse following ivosidenib monotherapy in IDH1-mutant relapsed or refractory AML. Blood Adv. 4 1894-1905 (2020).

178. Dohner, H. et al. Randomized, phase 2 trial of low-dose cytarabine with or without volasertib in AML patients not suitable for induction therapy. Blood 124, 1426-1433 (2014)

179. Zeidan, A. M. et al. Safety, efficacy and biomarker analysis of a phase 1b/ 2 study of onvansertib (ONV), a polo-like kinase 1 (PLK1) inhibitor, in combination with low-dose cytarabine (LDAC) or decitabine (DEC) in patients (pts) with relapsed/refractory acute myeloid leukemia (R/R AML). Blood 134, 230 (2019).

180. Riether, C. et al. Targeting CD70 with cusatuzumab eliminates acute myeloid leukemia stem cells in patients treated with hypomethylating agents. Nat. Med. 26, 1459-1467 (2020)

181. Daver, N. G. et al. A phase Ib/ll study of the CD123-targeting antibody-drug conjugate IMGN632 as monotherapy or in combination with venetoclax and/or azacitidine for patients with CD123-positive acute myeloid leukemia. Blood 134(Supplement_1), 2601, https://doi.org/10.1182/blood-2019-128501 (2019).

182. Agura, E., Gyurkocza, B. \& Nath, R. Targeted conditioning of lomab-B (1311anti-CD45) prior to allogeneic hematopoietic cell transplantation versus conventional care in relapsed or refractory acute myeloid leukemia (AML) 
preliminary feasibility and safety results from the prospective, randomized phase 3 sierra trial. Blood 132, 1017 (2018).

183. Vo, P. T. et al. Safety and efficacy of yttrium-90-labeled anti-CD45 antibody (90Y-DOTA-BC8) followed by a standard reduced-intensity hematopoietic stem cell transplant $(\mathrm{HCT})$ regimen for patients with refractory/relapsed leukemia or high-risk myelodysplastic syndrome (MDS). Blood 132, 1018(2018). 